\title{
MIPAS level 2 operational analysis
}

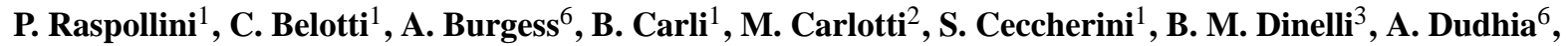 \\ J.-M. Flaud ${ }^{5}$, B. Funke ${ }^{8}$, M. Höpfner ${ }^{4}$, M. López-Puertas ${ }^{8}$, V. Payne ${ }^{6}$, C. Piccolo ${ }^{6}$, J. J. Remedios ${ }^{7}$, M. Ridolfi ${ }^{2}$, and \\ R. Spang ${ }^{7, *}$ \\ ${ }^{1}$ Istituto di Fisica Applicata "N. Carrara" (IFAC) del Consiglio Nazionale delle Ricerche (CNR), Firenze, Italy \\ ${ }^{2}$ Dipartimento di Chimica Fisica e Inorganica, University of Bologna, Bologna, Italy \\ ${ }^{3}$ Istituto di Scienza dell'Atmosfera e del Clima (ISAC) del Consiglio Nazionale delle Ricerche (CNR), Bologna, Italy \\ ${ }^{4}$ Forschungszentrum Karlsruhe GmbH, Institut für Meteorologie und Klimaforschung, Germany \\ ${ }^{5}$ Laboratoire Interuniversitaire des Systèmes Atmosphériques (LISA) CNRS/ Univ Paris 12 et 7, France \\ ${ }^{6}$ Atmospheric, Oceanic and Planetary Physics - Clarendon Laboratory - Oxford University, UK \\ ${ }^{7}$ Earth Observation Science, Department of Physics and Astronomy, University of Leicester, UK \\ ${ }^{8}$ Instituto de Astrofísica de Andalucía (CSIC), Granada, Spain \\ *now at: ICGI Forschungszentrum Jülich, Germany
}

Received: 15 February 2006 - Published in Atmos. Chem. Phys. Discuss.: 13 July 2006

Revised: 24 October 2006 - Accepted: 6 December 2006 - Published: 18 December 2006

\begin{abstract}
The MIPAS (Michelson Interferometer for Passive Atmospheric Sounding) instrument has been operating on-board the ENVISAT satellite since March 2002. In the first two years, it acquired in a nearly continuous manner high resolution $\left(0.025 \mathrm{~cm}^{-1}\right.$ unapodized) emission spectra of the Earth's atmosphere at limb in the middle infrared region. This paper describes the level 2 near real-time (NRT) and offline (OL) ESA processors that have been used to derive level 2 geophysical products from the calibrated and geolocated level $1 \mathrm{~b}$ spectra. The design of the code and the analysis methodology have been driven by the requirements for NRT processing. This paper reviews the performance of the optimized retrieval strategy that has been implemented to achieve these requirements and provides estimated error budgets for the target products: pressure, temperature, $\mathrm{O}_{3}, \mathrm{H}_{2} \mathrm{O}, \mathrm{CH}_{4}$, $\mathrm{HNO}_{3}, \mathrm{~N}_{2} \mathrm{O}$ and $\mathrm{NO}_{2}$, in the altitude measurement range from 6 to $68 \mathrm{~km}$.

From application to real MIPAS data, it was found that no change was needed in the developed code although an external algorithm was introduced to identify clouds with high opacity and to exclude affected spectra from the analysis. In addition, a number of updates were made to the set-up parameters and to auxiliary data. In particular, a new version of the MIPAS dedicated spectroscopic database was used and, in the OL analysis, the retrieval range was extended to reduce errors due to uncertainties in extrapolation of the profile outside the retrieval range and more stringent convergence criteria were implemented.
\end{abstract}

Correspondence to: P. Raspollini

(p.raspollini@ifac.cnr.it)
A statistical analysis on the $\chi^{2}$ values obtained in one year of measurements shows good agreement with the a priori estimate of the forward model errors. On the basis of the first two years of MIPAS measurements the estimates of the forward model and instrument errors are in general found to be conservative with excellent performance demonstrated for frequency calibration. It is noted that the total retrieval error is limited by forward model errors which make effectless a further reduction of random errors. However, such a reduction is within the capabilities of MIPAS measurements, which contain many more spectral signatures of the target species than what has currently been used. Further work is needed to reduce the amplitude of the forward model errors, so that the random error and the total error budget can be reduced accordingly.

The importance of the Averaging kernels for a full characterization of the target products is underlined and the equations are provided for their practical applications.

\section{Introduction}

The MIPAS (Michelson Interferometer for Passive Atmospheric Sounding) instrument (Fischer et al., 2000, 2006 ${ }^{1}$ ) is a Fourier transform infra-red spectrometer operating on board the environmental satellite ENVISAT launched by ESA on 1 March 2002. The first interferogram was measured

\footnotetext{
${ }^{1}$ Fischer, H., Birk, M., Blom, C., et al.: MIPAS: an instrument for atmospheric and climate research, Atmos. Chem. Phys. Discuss., in preparation, 2006.
}

Published by Copernicus GmbH on behalf of the European Geosciences Union. 
on 24 March 2002 and from July 2002 nearly continuous measurements were obtained for the first two years of satellite operation. Due to problems with the mirror drive of the interferometer, MIPAS ceased measurements at the end of March 2004. Operations with reduced maximum path difference, corresponding to both a lower spectral resolution and a shorter measurement time, were resumed in January 2005. This paper deals with the high spectral resolution measurements performed in the first two years of MIPAS operations, while the choices associated with the new measurement scenario with reduced spectral resolution implemented after January 2005 will not be discussed.

The instrument is designed to perform measurements during both day and night for the determination, on a global scale, of the vertical profile of the Volume Mixing Ratio (VMR) of several atmospheric species relevant for the characterization of the chemistry and climatology of the Earth's atmosphere. For this purpose, the spontaneous thermal emission of the atmosphere is observed in the middle infrared (from $685 \mathrm{~cm}^{-1}$ to $2410 \mathrm{~cm}^{-1}$ ), a region containing a large fraction of the black body emission of the atmosphere as well as features due to the vibrational spectra of most atmospheric constituents. In the first two years of operation the spectral resolution of MIPAS was $0.025 \mathrm{~cm}^{-1}$ (defined as the spacing between independent spectral elements of the unapodized spectrum). This spectral resolution is sufficient to distinguish the majority of individual atmospheric spectral lines at high altitudes, although not high enough to resolve their shape.

In order to obtain the vertical distribution of minor atmospheric constituents with good vertical resolution, limb viewing observations of the Earth's atmosphere were made with an elevation pointing that can be varied in tangent altitude from 5 to $210 \mathrm{~km}$ and with an Instantaneous Field of View (IFOV) of $3 \times 30 \mathrm{~km}^{2}$ (vertical height times across-track width, at $10 \mathrm{~km}$ tangent altitude).

A spectrum at the maximum spectral resolution is obtained in $4.5 \mathrm{~s}$. A limb sequence (or elevation scan) in the nominal observation mode is composed of 17 spectra that observe different tangent altitudes from 6 to $68 \mathrm{~km}$ and is acquired in $76.5 \mathrm{~s}$. During each orbit, MIPAS performs 75 limb sequences plus measurements for instrument calibration ("space" view and internal black-bodies for offset and gain determination).

The operational analysis, performed by ESA, requires a robust code with no choice delegated to the operator and with the capability of Near Real Time (NRT) operation, that is with a distribution of the products within three hours from the measurement time. These requirements drive the analysis methodology and therefore the results reported here reflect the analysis and quality of operational data rather than the ultimate limit of MIPAS capabilities.

While the level 1 analysis (Nett and Perron, 2002a; Nett et al., 2002b; Kleinert et al., 2006) determines, from the raw instrument data, the geolocated and calibrated spectra, the level 2 analysis determines, from level $1 \mathrm{~b}$ calibrated spec- tra, the geophysical parameters of interest. These include the pressure and temperature at tangent altitudes and the vertical profiles of six species selected to have highest priority, namely: $\mathrm{O}_{3}, \mathrm{H}_{2} \mathrm{O}, \mathrm{CH}_{4}, \mathrm{HNO}_{3}, \mathrm{~N}_{2} \mathrm{O}$ and $\mathrm{NO}_{2}$, in the altitude measurement range from 6 to $68 \mathrm{~km}$. These species are referred to as the target species.

The ESA operational level 2 code is based on a scientific prototype, named Optimized Retrieval Model (ORM) (Ridolfi et al., 2000), that was developed by an international consortium in the frame of an ESA Study. The mathematical complexity of the inversion problem, combined with the large amount of data to be processed for each limb sequence in NRT, determines the challenging and conflicting requirements of an accurate and time efficient retrieval. An optimized retrieval strategy and a series of mathematical and physical optimizations were implemented in the ORM code.

The ORM code is also the prototype of the Off-Line (OL) level 2 processor that is used for the re-analysis of the measurements with improved geolocation and set up of the level 1 and level 2 chain. In the OL analysis the computing time constraint is relaxed by a factor of 2 in CPU processing terms allowing more iterations per retrieval and an extended height range to be employed.

Given the novelty of the technical solutions implemented in the ORM, the assumptions and approximations adopted in this algorithm are critically discussed here in the light of MIPAS performance during the first two years of measurements and a review of the total error budget is provided.

The results of inter-comparison of MIPAS operational data with correlative measurements will not be reported in this paper, but in dedicated papers in the same Special Issue.

This paper is also intended to provide a summary of the most important information that is necessary to the potential users of NRT and OL level 2 MIPAS operational products provided by ESA and refers, in particular, to V4.61 and V4.62 of MIPAS OL products. In Sect. 2 the main features of the ORM are summarized, while the latest upgrades in both level 2 code and auxiliary data are described in Sect. 3. The different error sources in the forward model that affect accuracy of retrieved profiles are critically reviewed in Sect. 4 with the objective of assessing the correctness of the current estimation of the individual components. Section 5 provides the recipe for the computation of the total error budget indicating also where quantitative information of the different components can be found. Averaging Kernels of the retrieved profiles are shown in Sect. 6. An assessment of the ORM performances, based on a statistical analysis of one year of measurements, is made in Sect. 7, and finally, in Sect. 8, some examples of ORM products are shown.

\section{Summary of ORM features}

A detailed description of the ORM code is provided in Ridolfi et al. (2000), where the pre-flight performances 
obtained with simulation tests are also provided. For the benefit of the reader the main features of the ORM code are herewith briefly recalled.

The retrieval algorithm is based on the non-linear leastsquare fit (Menke, 1984; Sivia, 1998; Kalman, 1976; Rodgers, 2000) and consists of the global fit of a theoretical forward model calculation $\boldsymbol{F}(\boldsymbol{p}, \boldsymbol{x})$ to the observations $\boldsymbol{y} . \quad \boldsymbol{F}(\boldsymbol{p}, \boldsymbol{x})$ simulates the observations starting from the quantities $x$ to be retrieved and from a set of instrumental and geophysical parameters $\boldsymbol{p}$, that are considered to be known. The solution is found with an iterative procedure that uses the Gauss-Newton method, modified according to the Levenberg-Marquardt (Levenberg, 1944; Marquardt, 1963) criterion, for the minimization of the $\chi^{2}$ function. The $\chi^{2}$ function is equal to:

$\chi^{2}=\boldsymbol{n}_{\text {iter }}^{T} \mathbf{V}_{n}^{-1} \boldsymbol{n}_{\text {iter }}$,

where $\boldsymbol{n}_{\text {iter }}=\boldsymbol{y}-\boldsymbol{F}\left(\boldsymbol{p}, \boldsymbol{x}_{\text {iter }}\right)$ is the vector of the residuals at iteration "iter", with $\boldsymbol{x}_{\text {iter }}$ equal to either the initial guess or the result of the current iteration, and $\mathbf{V}_{n}$ is the VCM (VarianceCovariance Matrix) of the observations.

At each iteration $\boldsymbol{x}_{\text {iter }}$ is given by:

$$
\begin{aligned}
\boldsymbol{x}_{\text {iter }}= & \boldsymbol{x}_{\text {iter-1 }}+\left(\mathbf{K}_{\text {iter-1 }}^{T} \mathbf{V}_{n}^{-1} \mathbf{K}_{\text {iter-1 }}+\lambda \mathbf{I}\right)^{-1} \\
& \mathbf{K}_{\text {iter-1 }}^{T} \mathbf{V}_{n}^{-1} \boldsymbol{n}_{\text {iter-1 }}
\end{aligned}
$$

where $\mathbf{K}_{\text {iter }-1}=\frac{\partial \boldsymbol{F}\left(\boldsymbol{p}, \boldsymbol{x}_{\mathrm{iter}-1}\right)}{\partial x_{\text {iter }-1}}$ is the Jacobian of the simulated radiance relative to the profile $\boldsymbol{x}_{\text {iter-1 }}$ and $\mathbf{I}$ is the identity matrix. The Marquardt factor $\lambda$, that at each iteration is either increased or decreased depending on whether the $\chi^{2}$ function increases or decreases, reduces the amplitude of the iteration steps and ensures that convergence is reached.

At the last iteration, the final value of lambda is expected to be small, so that its effect on the retrieval error can be neglected. In this case, we can define the solution matrix of the inverse problem or gain matrix as:

$\mathbf{G}=\left(\mathbf{K}^{T} \mathbf{V}_{n}^{-1} \mathbf{K}\right)^{-1} \mathbf{K}^{T} \mathbf{V}_{n}^{-1}$

and the errors associated with the solution of the inversion procedure can be characterized by the VCM of $\boldsymbol{x}$ given by:

$\mathbf{V}_{x}=\mathbf{G} \mathbf{V}_{n} \mathbf{G}^{T}=\left(\mathbf{K}^{T} \mathbf{V}_{n}^{-1} \mathbf{K}\right)^{-1}$.

The MIPAS inversion process resulted to be sufficiently wellconditioned and regularization and a priori information are not necessary for a stable retrieval.

The retrieval strategy adopted to handle the multiplicity of unknowns and the redundancy of the data is based upon the following three corner stones:

1. Sequential retrieval of the species

The different unknowns are retrieved following a hierarchy of operations: first temperature and tangent pressures are retrieved simultaneously (henceforth denoted as the "p, T retrieval"), then VMR profiles of the target species are individually retrieved following the order of their reciprocal spectral interference, i.e.: $\mathrm{H}_{2} \mathrm{O}$ first, followed by $\mathrm{O}_{3}, \mathrm{HNO}_{3}, \mathrm{CH}_{4}, \mathrm{~N}_{2} \mathrm{O}$ and $\mathrm{NO}_{2}$. The simultaneous p, T retrieval (see e.g. Carlotti and Ridolfi, 1999) uses $\mathrm{CO}_{2}$ spectral features and exploits the hydrostatic equilibrium assumption that provides a relationship between temperature, pressure and geometrical altitude, the latter being determined by the engineering measurement of the pointing direction. Besides the target parameters, each retrieval also determines a frequency and altitude independent instrument zero-level offset and a frequency independent, altitude dependent absorption cross-section that models the atmospheric continuum and accounts for all the continuum-like emission effects that are not included in the line-by-line calculations.

2. Use of "microwindows"

The retrieval is performed using a set of narrow (less than $3 \mathrm{~cm}^{-1}$ width) spectral intervals, called "microwindows" (Dudhia et al., 2002a), that are selected as those intervals that contain the best information on the target parameters and are less affected by forward model errors, such as for instance uncertain spectroscopic data, interference of non-target species, Non-Local Thermal Equilibrium (NLTE) and line mixing effects.

3. Global fit analysis of the limb sequence

The global fit approach (Carlotti, 1988) is adopted for the retrieval of each vertical profile. This means that all the spectral data of a complete limb sequence are fitted simultaneously. The global fit provides a full exploitation of the measurements and a rigorous determination of the correlation between atmospheric parameters at the different altitudes.

The core and the most time consuming part of the retrieval code is the forward model. A self-standing subroutine of the code, called OFM (Optimized Forward Model) performs this calculation. OFM computes the atmospheric radiance measured by the spectrometer as the result of the radiative transfer in a non-uniform medium as well as of the instrument effects. The OFM also computes the Jacobians with respect to the retrieved quantities.

Features that are taken into account in the OFM are:

1. the effect of refractive index in the ray tracing of the optical path

2. Voigt profile for line shape modelling

3. use of a fine grid $\left(5 \times 10^{-4} \mathrm{~cm}^{-1}\right)$ in the spectral domain for a monochromatic modelling of radiative transfer

4. convolution of the atmospheric spectrum with the Instrument Line Shape (ILS) and IFOV of the instrument. 
On the other hand, NLTE effects, line mixing and pressure shifts have not been considered in the forward model, but are accounted for in the microwindow selection and in the error budget. The atmosphere is assumed to be horizontally homogeneous and in hydrostatic equilibrium.

Several mathematical and physical optimizations were studied to optimize the trade-off between accuracy and computing time. The main optimizations implemented in the OFM are:

1. definition of a sequence of operations that avoids the repetition of the same calculations and minimizes the number of memorized quantities;

2. use of cross-section look-up tables for each species, compressed with the Singular Value Decomposition method (Dudhia et al., 2002b);

3. use of a pre-defined subset of the fine grid points of the spectral domain (irregular grid) for the computation of the fine grid spectra;

4. Curtis-Godson approximation (Houghton, 1986) for cross section calculation in order to reduce the vertical segmentation of the atmosphere;

5. analytical IFOV convolution performed using a polynomial approximation of the tangent altitude dependence of the spectrum (polynomials from the 2 nd to the 4 th order are used going from high altitudes to low altitudes);

6. analytical computation of derivatives;

7. apodization of the spectrum with the Norton-Beer strong (Norton and Beer, 1977) function. This is done in order to reduce the contribution of side-lobes of the lines lying outside the microwindow and hence limit the size of the spectral range in which the spectrum is calculated (typically an extension of $0.175 \mathrm{~cm}^{-1}$ on both boundaries is considered) (Ceccherini et al., 2006 ${ }^{2}$ ).

Before ENVISAT was launched, the optimized forward model was validated against a Reference Forward Model developed at Oxford (Edwards, 1997). The retrieval code was tested both with self-consistency tests and against real measurements supplied by the MIPAS instrument on board of a stratospheric balloon (Friedl-Vallon et al., 2004), and by ATMOS (Abrams et al., 1996).

The exercise with data acquired by the balloon instrument, that required the adaptation of the code to a different observation geometry, was meant to identify the critical parts of the MIPAS NRT processor, in particular it highlighted the importance of the accuracy in ILS definition, the importance of

\footnotetext{
${ }^{2}$ Ceccherini, S., Belotti, C., Carli, B., and Raspollini, P.: The use of apodization in quantitative spectroscopy, Opt. Lett., submitted, 2006.
}

the used set of microwindows and the influence of non-target species on the retrieval.

Concerning the comparison with ATMOS measurements (Piccolo et al., 2004), an agreement within the total error was obtained between MIPAS Level 2 NRT algorithm and Jet Propulsion Laboratory (JPL) profiles (Irion et al., 2002). Furthermore, the level 2 analysis was used as a diagnostic tool to infer some instrumental effects and to identify the most important sources of systematic errors. The main effects on the retrieval were attributed to spectroscopic errors and non-optimal selection of the spectral microwindows.

Both the forward and the retrieval model have participated in the inter-comparison study with other retrieval codes in the frame of the AMIL2DA project (von Clarmann et al., $2003 \mathrm{c}$, a). The conclusions of this study were that the results of OFM and ORM agree within the predicted error margins with those provided by the other processors considered in the AMIL2DA project. Moreover, the blind test retrieval experiment highlighted that the retrieval code is capable of producing reliable results, in the sense that the discrepancies between retrieved and true (assumed) profiles are statistically consistent with the profile error bars determined on the basis of analytical error propagation.

The performance of the MIPAS level 2 processor strongly depends on the used auxiliary data. For this reason in the following sub-sections the databases of the auxiliary data used by MIPAS level 2 processor are described.

\subsection{Spectroscopic database}

A dedicated spectroscopic database is used for the analysis of MIPAS measurements. It was based on the HITRAN96 database (Rothman et al., 1998, and http://www. hitran.com) with improvements obtained through new laboratory studies and new calculations. A first version of the MIPAS database, named "mipas_pf2.0", included updates for the $\mathrm{HOCl}, \mathrm{HNO}_{3}, \mathrm{O}_{3}, \mathrm{NO}_{2}, \mathrm{CH}_{4}$ and $\mathrm{H}_{2} \mathrm{O}$ molecules that were validated performing comparisons between atmospheric simulated spectra and atmospheric spectra measured by the ATMOS experiment that flew on the Shuttle (Piccolo et al., 2004). A new version of the spectroscopic database, named mipas_pf3.1 (Flaud et al., 2003a) was released after the launch and it was used for both the NRT and OL re-processing (starting with V4.59 of the ESA Instrument Processing Facility). Details of this version are provided in Sect. 3.2.

\subsection{Reference atmosphere database}

The MIPAS processing requires a climatology of profiles that are used both to define the VMR of those species that interfere with the analysed spectrum, but are not currently retrieved (interfering species), and for the determination of the initial guess of the retrieved species. The initial guess of the retrieved species is obtained as a weighted mean of the 
profile retrieved at the previous limb sequence, if it exists, the ECMWF profile and a seasonal climatological profile.

This seasonal climatology, referred to as the initial guess climatology (IG2), was constructed (Remedios, 1999) for temperature and 36 species on a $1 \mathrm{~km}$ grid from 0 to $120 \mathrm{~km}$, mainly using a combination of observed satellite data (particularly from the UARS Reference Atmosphere project), tropospheric profiles from the MOZART model (Hauglustaine et al., 1998) and stratospheric profiles from the SLIMCAT model (Chipperfield, 1999). The seasons are three month periods, with mid-seasons of January, April, July and October arranged in six latitude bands per season $\left(0-20^{\circ}, 20-65^{\circ}, 65-\right.$ $90^{\circ}$ in each hemisphere). The trace gases included are: $\mathrm{CO}_{2}$, $\mathrm{O}_{3}, \mathrm{H}_{2} \mathrm{O}, \mathrm{CH}_{4}, \mathrm{~N}_{2} \mathrm{O}, \mathrm{HNO}_{3}, \mathrm{CO}, \mathrm{NO}_{2}, \mathrm{~N}_{2} \mathrm{O}_{5}, \mathrm{ClO}, \mathrm{HOCl}$, $\mathrm{ClONO}_{2}, \mathrm{NO} \mathrm{HNO}_{4}, \mathrm{HCN}, \mathrm{NH}_{3}, \mathrm{CFC} 11, \mathrm{CFC} 12, \mathrm{CFC} 14$, CFC22, $\mathrm{CCl}_{4}, \mathrm{COF}_{2}, \mathrm{H}_{2} \mathrm{O}_{2}, \mathrm{C}_{2} \mathrm{H}_{2}, \mathrm{C}_{2} \mathrm{H}_{6}, \mathrm{OCS}, \mathrm{SO}_{2}, \mathrm{SF}_{6}$, CFC13, CFC21, CFC113, CFC114, CFC115, $\mathrm{CH}_{3} \mathrm{Cl}, \mathrm{N}_{2}$ and $\mathrm{O}_{2}$.

In addition, five standard atmospheres were also produced to represent typical mean yearly profiles for Tropical $\left(0^{\circ}\right)$, Mid-latitude Day $\left(45^{\circ}\right)$ and Mid-latitude Night $\left(45^{\circ}\right)$, and typical mean seasonal profiles for Polar Winter $\left(\geq 70^{\circ}\right.$ in winter vortex, nightime conditions), Polar Summer $\left(\geq 70^{\circ}\right.$ in summer sunlit conditions). The principal purpose of these atmospheres was for use in the development of cross-section look-up tables for the forward model, for microwindow selection and for error estimation. The standard atmospheres were also designed to capture the variability of the atmosphere and included one sigma, maximum and minimum profiles based on the data sources described for the IG2 atmosphere. The one sigma profiles were employed in the microwindow selection process (Sect. 2.3) and in the error estimation (Sect. 4) for the target species.

\subsection{Microwindow database}

Microwindows are subsets of the two-dimensional measurement domain of tangent altitude versus spectral channel. The selection algorithm is described in detail in Dudhia et al. (2002a), but, briefly, is based on minimizing the total error defined as the sum of the measurement error $\mathbf{V}_{x}$ (due to random noise, see Eq. 4) and various forward model errors:

$\mathbf{V}_{x}^{\mathrm{tot}}=\mathbf{V}_{x}+\sum_{i} \mathbf{V}_{x}^{i}$

where each $\mathbf{V}_{x}^{i}$ represents an independent forward model error. The individual sources of these errors will be discussed in Sect. 4.

For each of these errors an error spectrum $\delta \boldsymbol{y}_{i}$ is computed as the difference between the forward model calculations obtained for a nominal value of the parameter and for the parameter perturbed by $1 \sigma$. The VCM of the forward model error in the measurement domain is given by:

$\mathbf{V}_{y}^{i}=\left(\delta \boldsymbol{y}_{i}\right)\left(\delta \boldsymbol{y}_{i}\right)^{T}$, while the VCM in the retrieval domain is given by:

$\mathbf{V}_{x}^{i}=\mathbf{G V}_{y}^{i} \mathbf{G}^{T}$

where $\mathbf{G}$ is the Gain Matrix used in the retrieval (see Eq. 3).

In order to provide a single set of microwindows which can be used globally, the actual minimized quantity represents a combination of the total errors evaluated for the five standard atmospheres described in Sect. 2.2.

As well as determining the boundaries of the microwindow, the selection procedure allows spectral points within the microwindow to be "masked", i.e., excluded from the retrieval. This happens when the inclusion of the measurement would increase rather than decrease the total error or, in other words, the increase in forward model error outweighs the decrease in random error.

This "negative information" concept is a direct consequence of the retrieval choices. Effectively, the microwindow selection attempts to minimize the impact of forward model errors allowing for the fact that the retrieval itself, with the minimization of $\chi^{2}$, will only weight each measurement according to its random error contribution instead of the total error.

Figure 1 shows the locations of the microwindows used for the operational retrievals and compares them with the contribution of each species to the observed spectrum. The microwindows tend to be selected in spectral regions where the lines of the target molecule dominate. In Table 1 the complete list of nominal microwindows (i.e. those used in absence of both corrupted spectra and corrupted bands) are reported, together with their altitude range.

The retrieval is performed using a set of the "best microwindows", chosen according to an ad hoc criterion which attempted to retrieve the maximum information on the target species with the minimum CPU cost. In practise, the effect of including the CPU cost limited the total number of microwindows which were used rather than affecting the actual microwindows which were selected. There is a limit after which no further microwindow can be added which reduce the total error of the selected set (Dudhia, 2004 ${ }^{3}$ ). However, the number of microwindows used in the operational processing of MIPAS data lies well inside this limit. Indeed, the limits of available computing power impose a time efficiency selection criterion within which a balance between random errors and forward model errors is sought.

The small number of microwindows and the use of spectral masks imply that only a few percent of the total number of measurements in each spectrum are actually used in the operational retrieval.

\footnotetext{
${ }^{3}$ Dudhia, A.: Information Content of Limb Radiances from MIPAS, http://www.ecmwf.int/publications/library/do/references/ show?id=86384, 2004 .
} 


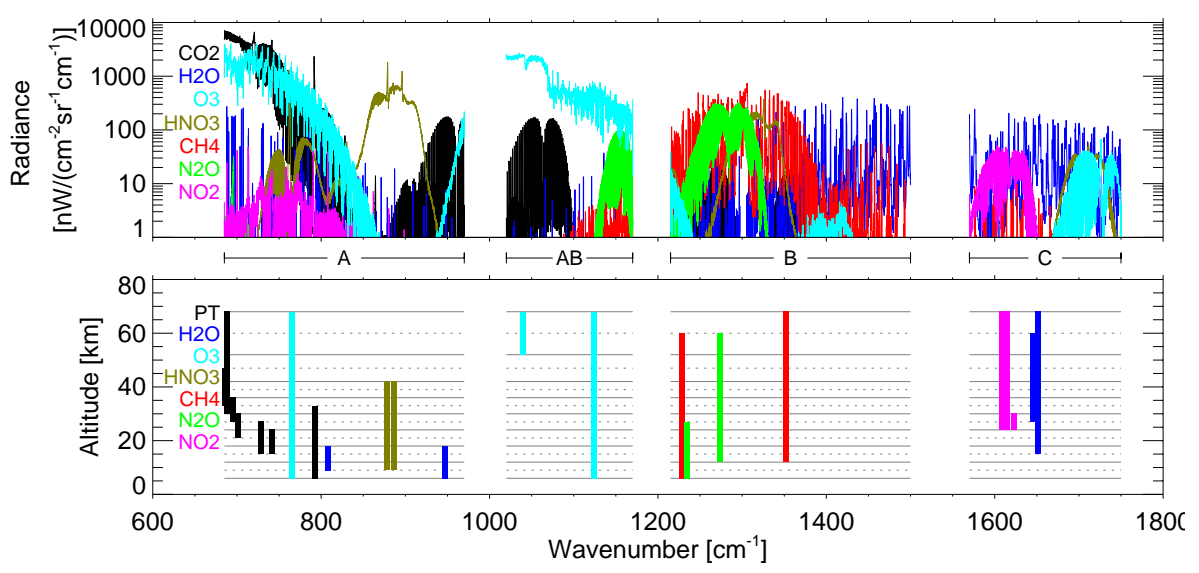

Fig. 1. The upper plot shows the major absorbing molecules in the MIPAS spectra for $21 \mathrm{~km}$ tangent altitude and the lower plot shows the spectral locations and tangent altitude range of the microwindows used for the MIPAS retrievals (note that the actual spectral width of each microwindow - maximum $3 \mathrm{~cm}^{-1}-$ is too narrow to be shown to scale on these plots). A, AB, B and C are the first 4 MIPAS bands.

\section{Post-launch upgrades}

The preparatory studies lasted for about seven years (Carli et al., 2004) and made possible the development of a quite reliable code. Indeed the analysis of the real MIPAS measurements indicated that only some minor upgrades in the level 2 processor were necessary. These have involved the pre-processor operations and the operating environment of the code defined by the input auxiliary data (including both retrieval settings and databases) and no change was made to the code itself. In the pre-processor a cloud filtering algorithm (see Sect. 3.1) was included to make the ORM more robust and the retrieved profiles more accurate.

For the input auxiliary data, a new version of the MIPAS dedicated spectroscopic database was released (see Sect. 3.2), a revised retrieval altitude range was adopted (see Sect. 3.3) and new convergence criteria were generated (see Sect. 3.4).

\subsection{Cloud filtering}

Shortly after launch, the new feature of the cloud filtering algorithm (Spang et al., 2004) was introduced in the preprocessor of MIPAS level 2 code. Clouds have a very wide spectral signature and affect the measured spectra at all the wavelengths that are transparent to emission from the atmospheric layer containing the clouds. Both low resolution (cloud continuum) and high resolution features (line distortions due to scattering) can be observed at the spectral resolution of MIPAS (Höpfner et al., 2002; Spang et al., 2004; Greenhough et al., 2005); spectral bands may also be observed for polar stratospheric clouds (Spang and Remedios, 2003; Höpfner et al., 2005). This implies that on the one hand cloud information can be extracted from MIPAS measurements, but on the other hand spectra containing clouds can spoil the quality of the trace gas retrieval. Hence, clouds with high opacity must be identified and flagged so that retrievals of trace gas concentration are performed above the cloud top height. An example of the global cloud top height map obtained from the analysis of 3 days of MIPAS measurements from 15 September 2002 is shown in Fig. 2. The highest cloud heights are found, as expected, in the tropical upper troposphere (cirrus) and in the Antarctic polar vortex (polar stratospheric clouds ). The polar stratospheric clouds in this period were the final ones observed prior to the unusually early break-up of the vortex in that year (see also Sect. 8).

For MIPAS operational processing, the ideal cloud filtering algorithm was one which would be fast, did not involve iterative retrievals (to save CPU time) and which classified cloud influences on measured spectra independently of the altitude of the cloud. Fortunately, the MIPAS spectral coverage allows this to be achieved very well using threshold tests applied to the ratio of the integrated signals in two microwindows (Spang et al., 2001, 2002), originally defined within what is Band A $\left(685-970 \mathrm{~cm}^{-1}\right)$ of the MIPAS instrument. Subsequent calculations with the Oxford Reference Forward Model identified useful cloud microwindow pairs in Band B $\left(1215-1500 \mathrm{~cm}^{-1}\right)$ and $\mathrm{D}\left(1820-2410 \mathrm{~cm}^{-1}\right)$; no such pairs could be determined in Bands AB $\left(1020-1170 \mathrm{~cm}^{-1}\right)$ and C $\left(1570-1750 \mathrm{~cm}^{-1}\right)$. As implemented for MIPAS processing, the cloud detection scheme is arranged hierarchically so that the primary cloud test, which operates between 6 and $45 \mathrm{~km}$, is performed with the Band A pair of microwindows. Forward calculations with the Oxford RFM were performed with the five standard atmospheres to identify thresholds for each cloud microwindow pair. These cloud indices were further tested against data from the Cryogenic Spectrometers and Telescopes for the Atmosphere (CRISTA) experiment to verify similar behaviour of the different microwindow pairs. Based on this information, clouds are flagged if the ratio 
Table 1. List of nominal microwindows.

\begin{tabular}{|c|c|c|}
\hline Microwindow label & Spectral range & Altitude range \\
\hline \multicolumn{3}{|c|}{$\mathrm{P}, \mathrm{T}$ retrieval } \\
\hline $\mathrm{PT}_{---} 039$ & $685.7000-685.8250 \mathrm{~cm}^{-1}$ & $33.0-47.0 \mathrm{~km}$ \\
\hline $\mathrm{PT}_{---} 001$ & $686.4000-689.4000 \mathrm{~cm}^{-1}$ & $30.0-68.0 \mathrm{~km}$ \\
\hline $\mathrm{PT}_{-\_-037}$ & $694.8000-695.1000 \mathrm{~cm}^{-1}$ & $27.0-36.0 \mathrm{~km}$ \\
\hline $\mathrm{PT}_{---038}$ & $700.4750-701.0000 \mathrm{~cm}^{-1}$ & $21.0-30.0 \mathrm{~km}$ \\
\hline $\mathrm{PT}_{---} 004$ & $728.3000-729.1250 \mathrm{~cm}^{-1}$ & $15.0-27.0 \mathrm{~km}$ \\
\hline $\mathrm{PT}_{---006}$ & $741.9750-742.2500 \mathrm{~cm}^{-1}$ & $15.0-24.0 \mathrm{~km}$ \\
\hline $\mathrm{PT}_{--} 002$ & $791.3750-792.8750 \mathrm{~cm}^{-1}$ & $6.0-33.0 \mathrm{~km}$ \\
\hline \multicolumn{3}{|c|}{$\mathrm{H}_{2} \mathrm{O}$ retrieval } \\
\hline H2O__002 & $807.8500-808.4500 \mathrm{~cm}^{-1}$ & $12.0-18.0 \mathrm{~km}$ \\
\hline $\mathrm{H} 2 \mathrm{O} \_-022$ & $946.6500-947.7000 \mathrm{~cm}^{-1}$ & $6.0-18.0 \mathrm{~km}$ \\
\hline H2O__007 & $1645.5250-1646.2000 \mathrm{~cm}^{-1}$ & $27.0-60.0 \mathrm{~km}$ \\
\hline H2O_-001 & $1650.0250-1653.0250 \mathrm{~cm}^{-} 1$ & $15.0-68.0 \mathrm{~km}$ \\
\hline \multicolumn{3}{|c|}{$\mathrm{O}_{3}$ retrieval } \\
\hline O3_-_021 & $763.3750-766.3750 \mathrm{~cm}^{-1}$ & $6.0-68.0 \mathrm{~km}$ \\
\hline O3 $3--013$ & $1039.3750-1040.3250 \mathrm{~cm}^{-1}$ & $52.0-68.0 \mathrm{~km}$ \\
\hline O3_--_001 & $1122.8000-1125.8000 \mathrm{~cm}^{-1}$ & $6.0-68.0 \mathrm{~km}$ \\
\hline \multicolumn{3}{|c|}{$\mathrm{HNO}_{3}$ retrieval } \\
\hline HNO3_001 & $876.3750-879.3750 \mathrm{~cm}^{-1}$ & $6.0-68.0 \mathrm{~km}$ \\
\hline HNO3_006 & $885.1000-888.1000 \mathrm{~cm}^{-1}$ & $6.0-42.0 \mathrm{~km}$ \\
\hline \multicolumn{3}{|c|}{$\mathrm{CH}_{4}$ retrieval } \\
\hline CH4__012 & $1227.1750-1230.1750 \mathrm{~cm}^{-1}$ & $6.0-60.0 \mathrm{~km}$ \\
\hline CH4__001 & $1350.8750-1353.8750 \mathrm{~cm}^{-1}$ & $12.0-68.0 \mathrm{~km}$ \\
\hline \multicolumn{3}{|c|}{$\mathrm{N}_{2} \mathrm{O}$ retrieval } \\
\hline N2O__012 & $1233.2750-1236.2750 \mathrm{~cm}^{-1}$ & $6.0-27.0 \mathrm{~km}$ \\
\hline N2O__001 & $1272.0500-1275.0500 \mathrm{~cm}^{-1}$ & $12.0-60.0 \mathrm{~km}$ \\
\hline \multicolumn{3}{|c|}{$\mathrm{NO}_{2}$ retrieval } \\
\hline NO2__001 & $1607.2750-1610.2750 \mathrm{~cm}^{-1}$ & $15.0-68.0 \mathrm{~km}$ \\
\hline NO2__003 & $1613.7250-1616.6000 \mathrm{~cm}^{-1}$ & $15.0-68.0 \mathrm{~km}$ \\
\hline NO2__013 & $1622.5500-1623.4750 \mathrm{~cm}^{-1}$ & $6.0-30.0 \mathrm{~km}$ \\
\hline
\end{tabular}

of radiances between microwindow $1\left(788.2-799.25 \mathrm{~cm}^{-1}\right)$ and microwindow $2\left(832.3-834.4 \mathrm{~cm}^{-1}\right)$ is less than $1.8 \mathrm{be}-$ tween $6 \mathrm{~km}$ and $45 \mathrm{~km}$; the upper limit of microwindow 1 can also be set to $796.25 \mathrm{~cm}^{-1}$ (Spang et al., 2002) which allows slightly more cloud sensitivity and is likely to be implemented for MIPAS in future processing. In case that band $A$ is not available, microwindows (1246.3-1249.1 $\left.\mathrm{cm}^{-1}\right)$ and (1232.3-1234.4 $\left.\mathrm{cm}^{-1}\right)$ in band $\mathrm{B}$ are used between 10 and $40 \mathrm{~km}$, with threshold 1.2. Finally, if neither band A nor band $\mathrm{B}$ are available, microwindows (1929.0-1935.0 $\mathrm{cm}^{-1}$ ) and $\left(1973.0-1983.0 \mathrm{~cm}^{-1}\right)$ in band D are used between 12 and $32 \mathrm{~km}$, with threshold 1.8 .
The determination of whether the line of sight is seriously affected by clouds and the consequent removal from the retrieval process of this line of sight (cloud filtering) was found to be an important operation. Firstly, it directly removed the anomalous mixing ratios that result from retrievals made with cloud-contaminated spectra. Secondly, it reduced the instabilities of the convergence process and improved the quality of MIPAS products above the clouds. At the same time, the scheme allows for the possibility that trace gas concentrations can still be retrieved for clouds or aerosol layers with low opacity in the infrared. 


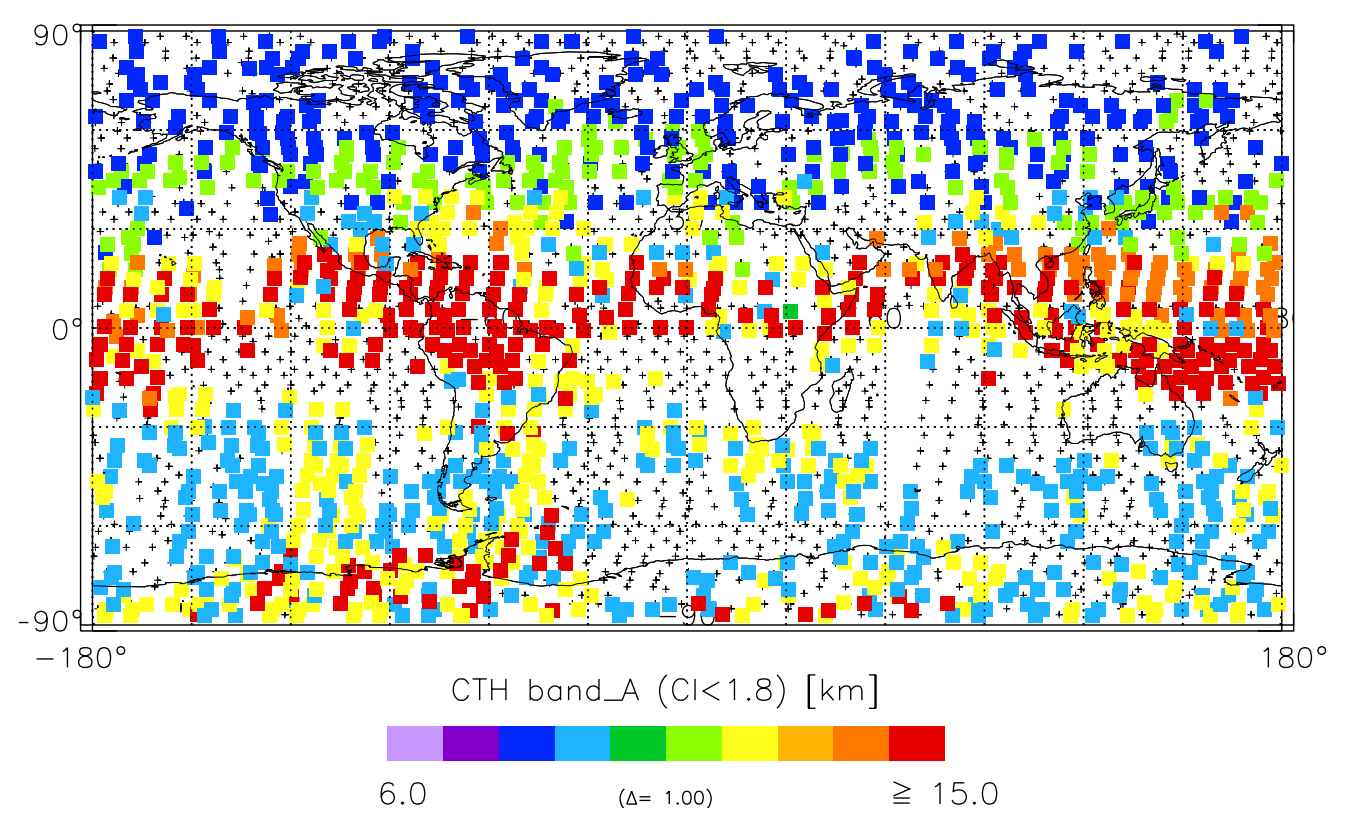

Fig. 2. Global cloud top height map from MIPAS data (OL data) for three days from 15 September 2002. Plus signs (+) indicate limb scans for which no clouds were found, i.e. the entire profile was cloud free. The highest cloud heights are found, as expected, in the tropical upper troposphere (sub-visible cirrus) and in the Antarctic polar vortex (polar stratospheric clouds or PSCs). The PSCs in this period were the final ones observed prior to the unusually early break-up of the vortex in that year. This figure was kindly provided by J. Greenhough.

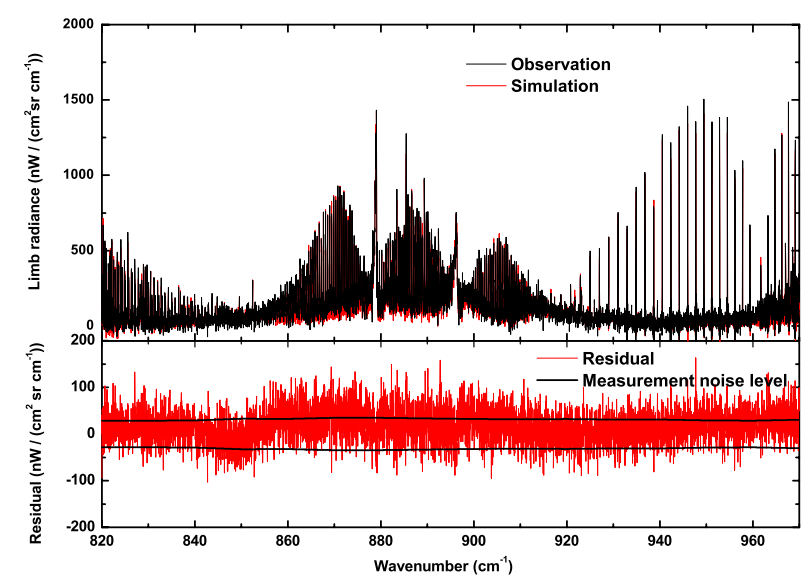

Fig. 3. Comparison between measurements and radiative transfer calculations in a spectra interval of band $\mathrm{A}$ that contains the $\mathrm{HNO}_{3}$ bands (two interacting $\mathrm{HNO}_{3}$ cold bands $v_{5}$ and $2 v_{9}$ centered at 879.109 and $896.448 \mathrm{~cm}^{-1}$, respectively, and two hot bands $v_{5}-v_{9}+v_{9}$ and $3 v_{9}-v_{9}$ located at 885.424 and $830.6 \mathrm{~cm}^{-1}$, respectively). Measured limb-radiances at $24 \mathrm{~km}$ tangent height (black) and corresponding simulation with the "mipas_pf3.1" spectroscopic line data (red) are shown in the top panel. The difference between the two spectra is shown by the red curve in the bottom panel, together with the specified measurement noise level in this spectral interval (black lines).

\subsection{Spectroscopic database}

After launch, version "mipas_pf3.1" (Flaud et al., 2003a) of the MIPAS dedicated spectroscopic database was released. It contains some improvements with respect to "mipas_pf2.0" based on both new laboratory spectroscopy and new calculations (Flaud et al., 2003b, c). The improvements concern the following molecules: $\mathrm{CO}_{2}, \mathrm{HNO}_{3}, \mathrm{CH}_{4}, \mathrm{NO}_{2}, \mathrm{O}_{3}$ and $\mathrm{COF}_{2}$.

In particular, the new spectroscopic data for $\mathrm{HNO}_{3}$ are responsible of a change of about $14 \%$ in the retrieved $\mathrm{HNO}_{3}$ VMR (Flaud et al., 2003a, b; Mencaraglia et al., 2006). From a comparison of the average $\mathrm{HNO}_{3} \chi^{2}$ obtained from the analysis of a whole orbit with both mipas_pf2.0 and mipas_pf3.1 versions of the spectroscopic database, it is found that the new database provides on average at all altitudes a reduction of $5 \%$ in the $\mathrm{HNO}_{3} \chi^{2}$ value.

In Fig. 3 the measured wide band spectrum relative to the spectral interval of band A between 820 and $970 \mathrm{~cm}^{-1}$ containing four $\mathrm{HNO}_{3}$ bands (two interacting $\mathrm{HNO}_{3}$ cold bands $v_{5}$ and $2 \nu_{9}$ centered at 879.109 and $896.448 \mathrm{~cm}^{-1}$, respectively, and two hot bands $v_{5}-v_{9}+v_{9}$ and $3 v_{9}-v_{9}$ located at 885.424 and $830.6 \mathrm{~cm}^{-1}$, respectively) is compared with the corresponding simulated spectrum (computed using the retrieved profiles and the "mipas_pf3.1" spectroscopic database) in the case of a limb measurement at $24 \mathrm{~km}$ tangent altitude. Also the residuals and the measured NESR (Noise Equivalent Spectral Radiance) are reported. Residuals are consistent with the NESR level with no strong feature larger 
than the noise, proving that a good agreement is obtained between measurements and forward model calculations also for wide-band spectra. Further information on the MIPAS spectroscopic database updates can be found in two technical reports (Flaud and Piccolo, 2001, 2003).

\subsection{Retrieval range}

In the altitude domain, ORM determines discrete values of the VMR in correspondence of the measured tangent altitudes (or of a subset of them). In the nominal measurement scenario MIPAS measurements extend from 6 to $68 \mathrm{~km}$, but the level 2 retrieval can be limited to a selected molecule dependent altitude range (retrieval range). For the first postlaunch operations the choice was made to limit the retrieval range to those altitudes in which good retrieval accuracy was found to be possible with pre-flight tests. Before the implementation of the cloud filtering, the preliminary retrieval range did not extend below $12 \mathrm{~km}$ because of the high probability of cloud occurrence below this altitude. Above the highest point and below the lowest point of the retrieval range the shape of the profile is assumed to be equal to that of the initial guess profile and the fit just applies a scaling factor that avoid discontinuities and preserves the shape of the initial guess profile. This may introduce a bias in the retrieved profile which depends on the assumed shape (see Sect. 4.2.2). If the assumed shape of the profile above the highest tangent altitude is different from that in the real atmosphere, then the retrieval tries to compensate for the error in the estimated slant column by attributing a higher or lower value to the retrieved concentration at the highest tangent point. This error can propagate to the lower tangent points with an amplitude that quickly damps out as the distance from the highest tangent altitude increases.

The profile below the lowest tangent point is observed through the tail of the IFOV on the low altitude side. For this case, differences between the assumed and real shape of the profile lead to an incorrect computation of the IFOV convolution for the lowest tangent altitude and hence to an error in the retrieved concentration for the lowest retrieved point.

Tests performed after the ENVISAT launch proved the criticality of the adopted extrapolation strategy. Moreover the analysis of real data highlighted the need to extend the retrieval range of $\mathrm{NO}_{2}$ at higher altitudes to account for its increase during the polar night.

In order to overcome the approximations introduced by the extrapolation strategy and also to exploit the downward extension to $6 \mathrm{~km}$ made possible by the use of cloud filtering, the strategy adopted for the definition of the retrieval range was changed to include all the points that are useful for improving the overall quality of the retrieval, independently of the quality attained at that specific altitude. The extension of the retrieval range does not imply that the added retrieved points are always useful geophysical data. As it will be explained in Sect. 6, indications on the usefulness of retrieved
Table 2. Altitude retrieval range for the different species in both NRT and OL products.

\begin{tabular}{ccc}
\hline & Retrieval range for NRT & Retrieval range for OL \\
\hline $\mathrm{PT}$ & $12-68 \mathrm{~km}$ & $6-68 \mathrm{~km}$ \\
$\mathrm{H}_{2} \mathrm{O}$ & $12-60 \mathrm{~km}$ & $6-68 \mathrm{~km}$ \\
$\mathrm{O}_{3}$ & $12-60 \mathrm{~km}$ & $6-68 \mathrm{~km}$ \\
$\mathrm{HNO}_{3}$ & $12-42 \mathrm{~km}$ & $9-42 \mathrm{~km}$ \\
$\mathrm{CH}_{4}$ & $12-60 \mathrm{~km}$ & $6-68 \mathrm{~km}$ \\
$\mathrm{~N}_{2} \mathrm{O}$ & $12-47 \mathrm{~km}$ & $6-60 \mathrm{~km}$ \\
$\mathrm{NO}_{2}$ & $24-47 \mathrm{~km}$ & $24-68 \mathrm{~km}$ \\
\hline
\end{tabular}

data can be provided by the Averaging Kernel Matrices. For instance it has been found that the additional points retrieved by the OL analysis for $\mathrm{H}_{2} \mathrm{O}$ and $\mathrm{NO}_{2}$ (namely 68,9 and $6 \mathrm{~km}$ for $\mathrm{H}_{2} \mathrm{O}$ and 68 and $60 \mathrm{~km}$ for $\mathrm{NO}_{2}$ ) are not expected to have a physical meaning and the values retrieved at these altitude points are reported as part of the MIPAS products for their mathematical value only (they are the values used by the retrieval for modelling the overall profile).

The extension of the retrieval range implies an increase in the processing time, and hence this improvement is only implemented in the OL products, while the NRT products were obtained using the preliminary retrieval range.

The retrieval ranges for the different species are listed in Table 2 for both the NRT and the OL cases.

\subsection{Convergence criteria}

The retrieval is performed through an iterative procedure aimed at reaching the minimum of $\chi^{2}$. The convergence criteria determine when the iterative procedure stops. Even if the problem is non-linear, the uniqueness of the solution is assured both from a dedicated definition of the initial guess profiles of the retrieval in order to start as close as possible to the solution and from the fact that the thick lines are discarded by the microwindow selection, so that the problem is moderately non-linear.

The convergence criteria adopted in the MIPAS level 2 processor are based on three conditions:

1. Linearity of the inversion problem. The maximum relative difference between the $\chi^{2}$ projection made at the previous iteration on the basis of the linearity assumption and the real $\chi^{2}$ is less than a pre-defined threshold $\mathrm{T}_{1}$.

2. Attained accuracy. The maximum relative variation (in two subsequent iterations) of the fitted parameters is less than a pre-defined threshold $\mathrm{T}_{2}$.

3. Computing time. Due to general computing time constraints in MIPAS level 2 processor, there is a maximum number of allowed iterations. 
Table 3. MIPAS forward model error sources and their amplitudes.

\begin{tabular}{|c|c|c|}
\hline Error Source & $1 \sigma$ & Name [3] \\
\hline \multicolumn{3}{|l|}{ Errors in Instrument Characterisation } \\
\hline Radiometric Gain & $\pm 2 \%$ & GAIN \\
\hline Spectral Calibration & $\pm 0.001 \mathrm{~cm}^{-1}$ & SHIFT \\
\hline Apodised ILS Width & $\pm 2 \%$ & SPREAD \\
\hline \multicolumn{3}{|l|}{ Errors in radiative transfer parameters } \\
\hline Profiles of the 6 target gases & $\pm 10 \%$ & [gas] \\
\hline Profiles of 22 unretrieved gases & Climatological St. Dev. & [gas] \\
\hline High Altitude Column & Climatological St. Dev. & HIALT \\
\hline Spectral database errors & [1] & SPECDB \\
\hline Error profiles of retrieved $\mathrm{p}$ and $\mathrm{T}$ & Random covariance [2] & PT \\
\hline \multicolumn{3}{|l|}{ Deficiencies in radiative transfer } \\
\hline Non-LTE effects & Modelled & NONLTE \\
\hline $\mathrm{CO}_{2}$ Line Mixing & Modelled & CO2MIX \\
\hline Horizontal Temperature gradients & $\pm 1 \mathrm{~K} / 100 \mathrm{~km}$ & GRA \\
\hline
\end{tabular}

[1] Based on assumed $1 \sigma$ accuracies in line position, strength and half-width relative to HITRAN 96 database (Flaud, private communications).

[2] VCM of the pT retrieval error.

[3] "Name" is used in the legends of Figs. 4 and 5.

The retrieval is stopped if one of the above three conditions is fulfilled, the convergence is reached if one of the first two conditions is fulfilled.

Tests performed after ENVISAT launch showed the criticality of the convergence criteria thresholds. If, in order to reach convergence with a small number of iterations, the thresholds used for convergence criteria are not sufficiently stringent, the convergence error, defined as the difference between the profile at convergence and the profile obtained after 10 iterations, may be comparable with the random error (it was empirically found that after 10 iterations the results of the retrieval does not change anymore).

In general with more stringent thresholds a greater number of iterations is needed, with a consequent increase in computing time. As a consequence, more stringent thresholds are used only for the computation of the OL products.

It has been observed that in order to reduce the convergence error, the convergence must be determined more by the criterion on the maximum variation of the retrieved quantities than by the criterion on linear variation of $\chi^{2}$. This is obtained by using in the OL auxiliary data very stringent $\mathrm{T}_{1}$ values. Typical $T_{1}$ values in NRT auxiliary data are less than 0.15 , while $\mathrm{OL} \mathrm{T}_{1}$ values are less than 0.02 . The NRT $\mathrm{T}_{2}$ values are 0.01 , while $\mathrm{OL} \mathrm{T}_{2}$ values are less than 0.2 . Furthermore, the maximum number of iterations was reduced to 8 in the OL case from the value of 10 in the NRT case, since it was verified that typically 3-6 iterations are sufficient to reach convergence. Scans that do not reach convergence in 8 iterations are generally bad, and it was decided not to waste time with further iterations.

The convergence criteria adopted for OL products make the convergence error small enough to be neglected in the total error budget (typically the convergence error is a factor 3 lower than the random error in this case), while in NRT products the convergence error may be comparable with the random error.

\section{Assessment of total error budget of retrieved profiles}

As shown in Eq. (5), the total error budget of the retrieved profiles includes two contributions: the measurement error and the forward model errors.

The measurement error is due to the mapping of random radiometric noise into the retrieved profiles. The VCM $\mathbf{V}_{x}$ of the noise error is computed by ORM using Eq. (4). The VCM of the observations $\mathbf{V}_{n}$, that appears in that formula, is calculated using the radiometric noise (expressed in terms of Noise Equivalent Spectral Radiance NESR) provided by level 1 processor, and takes into account the correlation between spectral points due to the apodization process.

The forward model errors are due to uncertainties in instrument characterization and in input parameters of the radiative transfer, as well as to approximations in the forward model itself. The forward model errors are determined on the basis of a priori estimates of these uncertainties. The 


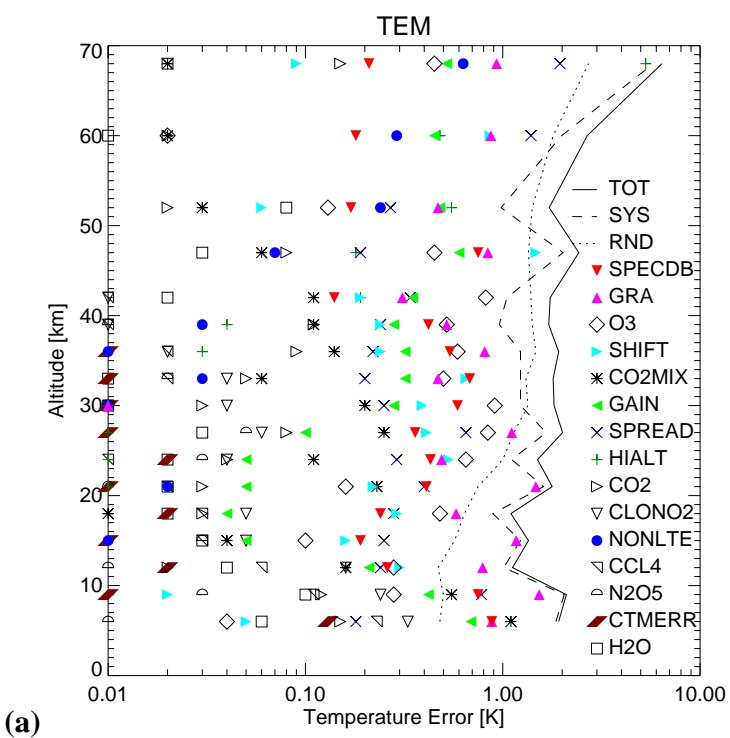

(b)
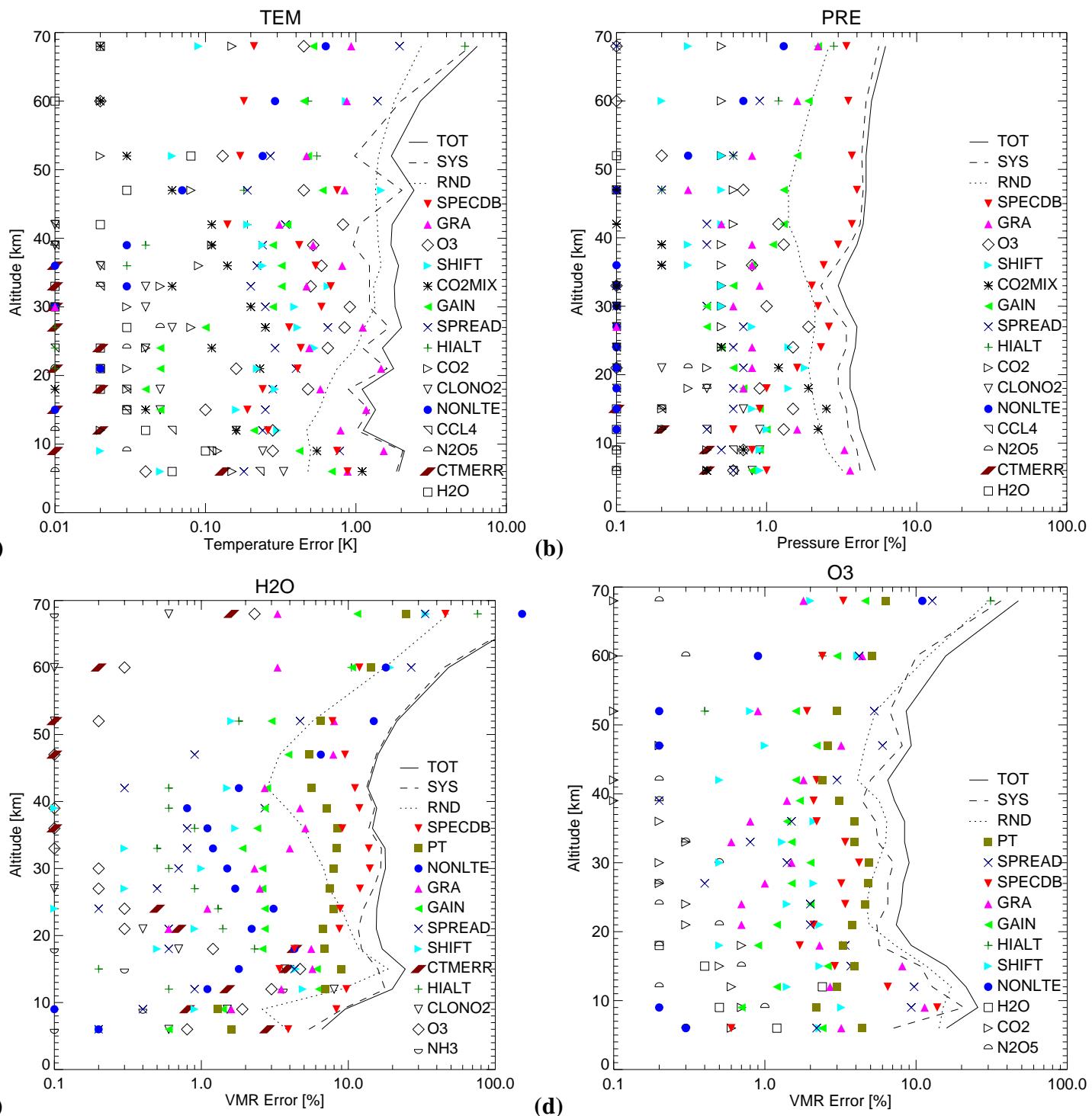

Fig. 4. Error budgets for the MIPAS retrievals of temperature, pressure, water vapour and ozone computed for midlatitude daytime conditions in the case of OL retrieval. The solid line is the Total Error (or Accuracy), represented by the rootmeansquare of the Random Error (or Precision), shown as the dotted line, and the forward model errors, shown as the dashed line. The forward model error is itself the rootmeansquare of the various components shown by different symbols (see Table 3 for details).

sources of forward model errors which have been considered are listed in Table 3 together with their assumed amplitudes.

The square roots of the diagonal elements of all matrices involved in Eq. (5) for mid-latitude day-time conditions and OL retrievals are shown for the various MIPAS products in Figs. 4 and 5. The random noise varies from measurement to measurement. The values shown in Figs. 4 and 5 (denoted by RND) are based on in-flight values of NESR from orbit 2081.

From these figures it emerges that the forward model error contribution is comparable with the measurement error. As the forward model errors are better understood they can be reduced and a new microwindow selection can be per- formed for a similar reduction of the measurement error and of the total error budget. A significant amount of work is in progress in this direction. Presently the total error is larger than the proposed objectives of the mission and significantly larger than what can be potentially obtained from the wide spectral coverage of MIPAS measurements.

In this section the results of the analysis performed with ORM to assess the correctness of the a priori estimates of the errors, as well as findings from other external tests, are reviewed in order to verify the estimation of each error component of the total error budget. 
(a)
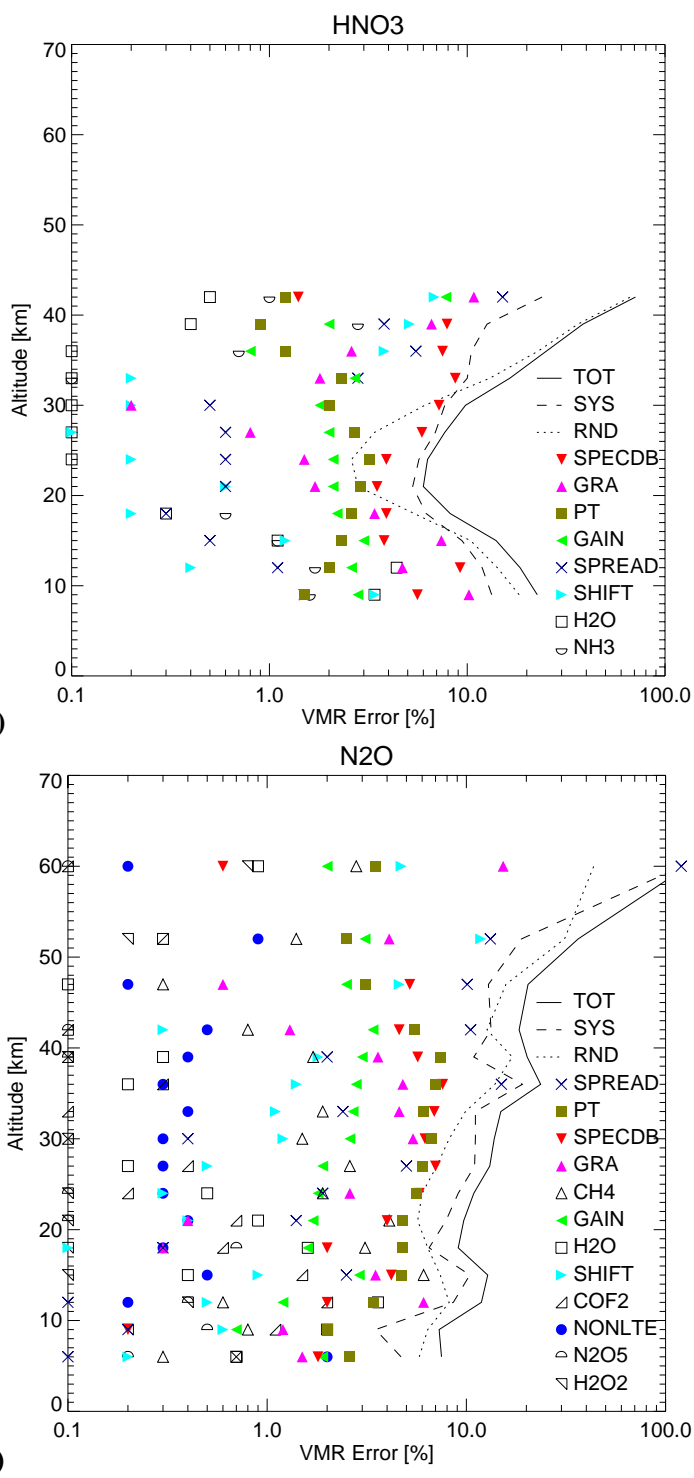

(b)
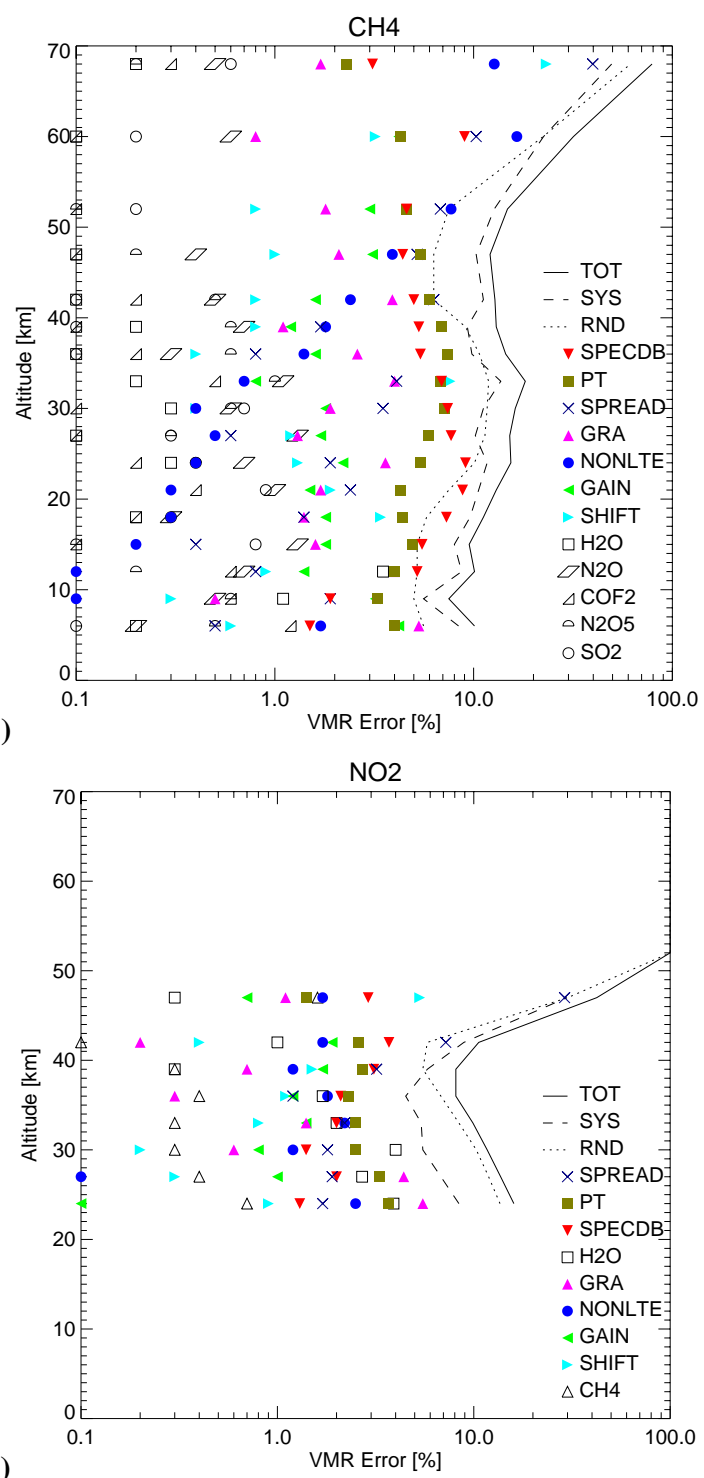

Fig. 5. As Fig. 4, showing retrieval error budgets for nitric acid, methane, nitrous oxide and nitrogen dioxide.

\subsection{Error in instrument characterization}

Instrument effects are modelled in the forward model and approximations in this model can be a source of error. ORM has the capability of retrieving, together with the target parameters, also some parameters related to instrument effects such as frequency calibration, width of ILS and radiometric calibration. The retrieval of these non-target parameters has allowed a validation of the instrument characterization and an assessment of the correctness of instrument error estimates.

\subsubsection{Radiometric gain}

Radiometric accuracy is crucial because it directly affects the accuracy of temperature and VMR retrievals. The high correlation between radiometric gain and retrieved quantities makes it difficult to extract information on radiometric accuracy from the level 2 analysis. Investigations performed on dedicated measurements for the instrument characterization have proved that the radiometric errors are within the specifications of MIPAS instrument performance (Birk and Wagner, 2002; Nett et al., 2002b). However, tests performed on the measured integrated radiance highlighted for some bands the presence of an oscillation in the gain calibration. The oscillation is correlated with the forward/reverse direction of 
the interferometer sweep and is more evident in those bands in which a non-linearity correction has to be applied to the detectors. This oscillation, even if it lies within the requirements, seriously affects the level 2 analysis because it enhances the anti-correlations that exist between retrieved values at contiguous altitudes and induces an oscillation in retrieved profiles. Forward-reverse oscillations have been significantly reduced with an improved non-linearity correction in level 1 processor and the residual effects that are present in the radiance cause an error in the retrieved profiles that is smaller than the mean random error.

In conclusion, the investigations on radiometric accuracy have shown that the instrument performance is within the requirements, and also the amplitude attributed to the radiometric gain error component, which is based on the requirements, is correct.

\subsubsection{Spectral calibration}

Tests performed by ORM to validate the spectral frequency calibration of level 1 processing were made with the fit of a frequency dependent and altitude independent frequency scaling factor for all target species. From the analysis of the frequency dependence of the frequency scaling factor it was found that, while level 1 processor assumes that the frequency dependence can be modelled with a slope, the dependence on frequency is better modelled by a slope and a quadratic term. This systematic difference is now corrected in the level 2 pre-processor.

Concerning the time dependence of the frequency scaling factor retrieved by ORM, Fig. 6 shows its deviation from unity as a function of the limb sequence index for a full orbit. This plot was obtained after the correction of the frequency dependence described above. The curves in the plot are grouped with different colours according to the number of spectral points used for the retrieval. Since a larger number of spectral points provide a better accuracy, the scattering of the retrieved values is reduced when more points are used (see blue curves). The spread of the curves (about 3 parts in $10^{7}$ ) gives an idea of the attained frequency calibration accuracy, that is better than the MIPAS requirement (equal to $\left.1 \times 10^{-6} \mathrm{~cm}^{-1}\right)$. A degradation of the performance is visible only in the first 3 scans of the orbit, characterized by large values of the frequency scaling factor. This is due to the use in level 1 processor of an approximated default calibration for those sequences that are analysed before the first frequency calibration measurement is made.

On the basis of these tests, we can conclude that the amplitude attributed to this error component $(\Delta \sigma / \sigma=0.001)$ is conservative. However, as it can be observed in Figs. 4 and 5 , this error is usually a small contribution to the total error budget. Indeed a frequency shift causes both a positive and a negative residual of the two sides of a line, which increases the value of the $\chi^{2}$, but has only a small effect on the value of the fitted intensity.

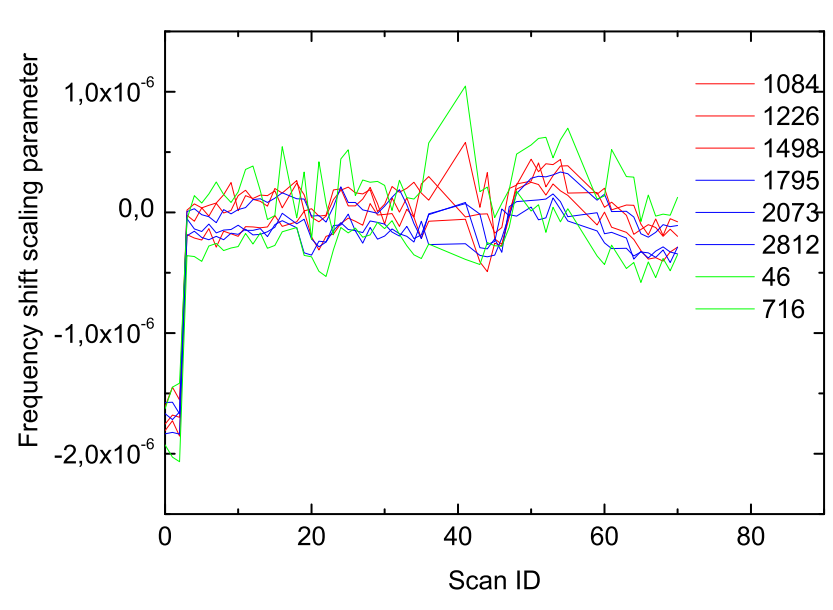

Fig. 6. Fit of the frequency scaling factor as a function of the scan identification number (ID) for different sets of spectral points (depending on the analysed species and on the measurement band). Different colours are used for the fits that use few points (green curves), medium number of points (red curves) and large number of points (blue curves).

\subsubsection{Width of the apodized instrument line shape}

The Apodized Instrument Line Shape (AILS) is equal to the convolution of the ILS determined by the level 1 processor with the apodization function adopted in the level 2 analysis. The width of the AILS is dominated by the width of the apodization function, but a correct modelling of the ILS is important because a small error in the ILS, even if it does not change significantly the AILS and the residuals, can cause a large variation of the $\chi^{2}$. This is explained by the fact that correlations are taken into account for the calculation of the $\chi^{2}$ (see Eq. 1) and the errors caused by the ILS, even if damped by the operation of apodization, are properly determined.

The ORM is able to fit a band dependent and altitude independent ILS broadening correction. A broadening correction that is practically equal to zero is retrieved. This, together with the low values of the $\chi^{2}$, indicates that the ILS width is determined in level 1 with an accuracy of about $0.2 \%$. On the other hand a different result is obtained from a statistical analysis of the observed residuals, performed using the first two years of MIPAS measurements. In general this analysis consists in the fit of the residuals with the error spectra associated to each error and is named Residual and Error Covariance (REC) analysis (Dudhia, 2002). For the analysis of the ILS width error, the residuals are fitted with the second derivative of the spectrum and a correlation of about $2 \%$ between the residual and the second derivative of the spectrum is found. This empirically detected second derivative error is referred to as ILS width error even if causes different from ILS modelling can also be responsible for it. Further investigations are on-going. 


\subsection{Errors in radiative transfer parameters}

Investigations have been made to check the correctness of the uncertainties in the input parameters of the forward model with respect to their assumed variability. Results are summarised below.

\subsubsection{Interfering species}

Each target species is retrieved by ORM with an individual fit in which all other interfering species are known parameters. For the interfering target species, whenever available, the profile retrieved in the previous fit is used and, for all other interfering species, the assumed profile is given by the IG2 climatological database described in Sect. 2.2.

The uncertainties associated with the climatological profiles are the climatological 1- $\sigma$ variabilities taken from extreme concentration profiles (Remedios, 1999) for each of the standard conditions (tropical, mid-latitude day/night, polar winter and polar summer). Concerning the contaminant target species, which are retrieved by MIPAS in a prior retrieval step, a total retrieval error of $10 \%$ is assumed.

The errors induced by the interfering species were verified with the REC analysis which has highlighted that the profile of some non-target species $\left(\mathrm{COF}_{2}, \mathrm{HOCl}, \mathrm{H}_{2} \mathrm{O}_{2}\right.$ and $\mathrm{NH}_{3}$ ) differs from the climatological profile by more than the climatological variability and the variability of some other non-target species $\left(\mathrm{HCN}, \mathrm{HNO}_{4}\right)$ is underestimated. However, none of these deviations affects significantly the error budget. An upgrade is probably needed for the variability of $\mathrm{SO}_{2}$ : the REC analysis indicates $\mathrm{SO}_{2}$ variability much smaller than assumed by the climatological variability. This is due to climatological variability allowing for volcanic activity whereas during MIPAS operation there has been no significant eruption affecting stratospheric $\mathrm{SO}_{2}$. A reduction of this climatological variability by a factor 10 is recommended since it is currently a significant component of the $\mathrm{CH}_{4}$ error budget.

4.2.2 Shape of VMR and temperature profiles outside of the retrieval range

As described in Sect. 3.3, ORM assumes a fixed-shape of the atmospheric profile above the highest and below the lowest retrieval levels. The error due to this approximation has been recently included in the total error budget (see Figs. 4 and 5 and website http://www-atm.physics.ox.ac.uk/group/ mipas/err) and is a significant error component at high altitudes. The $\mathrm{NO}_{2}$ retrieval is particularly affected by this error, due to the diurnal variability of this species and to the large variability of the profile with season and latitude. The extension of the retrieval range, implemented in the OL products, effectively reduces this error.

The error due to the assumption of a fixed shape of atmospheric profile outside the retrieval range is calculated assuming that the "true" profile can deviate by climatological variability. No significant error has been highlighted in the assumed climatological variability of the target species at high altitudes, and hence this error is now adequately accounted for.

\subsubsection{Spectroscopic database errors}

The spectroscopic database errors are due to uncertainties in the strength, position and width of infrared emission lines. These errors have been estimated using the available experimental data and/or calculations, but it is worth stressing that this is a difficult problem given the fact that in the papers reporting spectroscopic parameters a real estimation of the errors is not always performed. The spectroscopic error estimates reported in Figs. 4 and 5 are based on uncertainty parameters relative to previous versions of the spectroscopic database. In the mipas_pf3.1 release of the MIPAS dedicated spectroscopic database these errors have been reduced. Hence the unchanged estimates of the spectroscopic errors reported in Figs. 4 and 5 are most likely conservative.

\subsubsection{Temperature and tangent pressure propagation error}

The pointing of the instrument is a very critical quantity in the level 2 analysis of limb measurements. In order to compensate for bias angles and harmonic pointing variations during the orbit, the Line-of-Sight is determined with in-flight calibrations made viewing at stars. Tests performed after launch (von Clarmann et al., 2003b) showed that in the first few months of operations the MIPAS engineering tangent altitude had a bias of up to $3 \mathrm{~km}$, but after an upgrade of the MIPAS pointing system the bias was reduced within the accuracy specification, i.e. $900 \mathrm{~m}$. The short term pointing stability, during one interferometer sweep of $4 \mathrm{~s}$ duration, was verified to be better than $80 \mathrm{~m}$ (Ridolfi, 2005).

In order to further reduce the effect of the instrument pointing bias on retrieved profiles, the tangent pressures are fitted as part of the $\mathrm{p}, \mathrm{T}$ retrieval. The short term pointing stability is exploited to improve the $\mathrm{p}, \mathrm{T}$ retrieval with the hydrostatic equilibrium constraint that is obtained with the altitude increments of the engineering pointing. The retrieval of the tangent pressure is estimated to have an accuracy of about $1 \%$ which corresponds to an altitude uncertainty of about $70 \mathrm{~m}$.

Nevertheless, the altitude grid, that is derived from the pressure grid using the hydrostatic equilibrium and the lowest tangent altitude as a starting point, is affected by a total error larger than the retrieved tangent pressure. Indeed the large bias $(3 \mathrm{~km}$ in the early measurements and $900 \mathrm{~m}$ in the recent measurements) in the absolute value of the engineering tangent altitude induces a shift in the whole tangent altitude grid. For this reason it is preferred to represent the retrieved profiles as a function of pressure grid, which is an accurate retrieved quantity, and not as a function of the 
altitude grid, which is affected by the bias of the engineering pointing.

A pointing jitter was also detected in level 1 analyses of the raw full size interferogram ${ }^{4}$. The signal modulation that the jitter introduces in the interferogram is expected to generate some fake lines that in spectroscopy are called "ghosts". Tests made on the measured spectra have shown that no ghost is visible above the measurement noise and, because of the random phase of this jitter, even averaged spectra do not show the effect. This error is being monitored, but, because of its small amplitude, is presently not included in the forward model error budget.

Tangent pressure and temperature, which are simultaneously retrieved because of the large correlation that exists between the two parameters, are an important cause of error in VMR retrievals.

These errors are computed a posteriori using the propagation matrix $\mathbf{E}$ providing the mapping of $\mathrm{p}, \mathrm{T}$ error on the retrieved VMR profiles:

$\mathbf{V}_{x P T}=\mathbf{E} \mathbf{V}_{P T} \mathbf{E}^{T}$,

where $\mathbf{V}_{x P T}$ is the VCM of the $\mathrm{p}, \mathrm{T}$ induced error on the VMR profile and $\mathbf{V}_{P T}$ is the VCM of the p,T retrieval. In general this operation has to be performed for both the measurement error and the forward model components of $\mathrm{p}, \mathrm{T}$ error. In Figs. 4 and 5 only the measurement error is included in the estimate of this error component.

In general matrix $\mathbf{E}$ depends on the set of microwindows used for VMR retrievals and on the atmospheric status (temperature and VMR profiles). However, it has been verified (Raspollini and Ridolfi, 2000) that in practice the dependence on the atmospheric status can be neglected.

A set of pre-tabulated propagation matrices has been computed for the mid-latitude standard atmosphere for the nominal set of microwindows (see also Sect. 5).

\subsection{Deficiencies in the radiative transfer model}

The forward model computation is the most time consuming part of the retrieval code. Time efficiency is obtained by means of both physical and mathematical optimizations, as well as the use of microwindows that reduces the effect of some deficiencies in the forward model.

The results of the tests aimed at verifying the negligible effect of these deficiencies are discussed below.

\subsubsection{Line-mixing}

Line mixing (Edwards and Strow, 1991; Rosenkranz, 1975) corresponds to the deviation of the measured line shape from the Voigt function, occurring when collisions between a radiating molecule and the broadening gas molecules cause the transfer of population between different ro-vibrational states.

\footnotetext{
${ }^{4} \mathrm{M}$. Birk, private communications
}

Line mixing affects especially the Q-branches where transitions between ro-vibrational energy levels closer than $\mathrm{K}_{B} \mathrm{~T}$ ( $\mathrm{K}_{B}$ is the Boltzmann constant, $\mathrm{T}$ is the temperature) are packed together. The most apparent effect of line-mixing is a reduction of the cross-section in the wings of the branch.

Line mixing is neglected in the forward model, but the error of this approximation is minimized by the microwindow selection.

An investigation was performed on four microwindows selected for $\mathrm{p}, \mathrm{T}$ retrieval for which the line-mixing error spectra mostly approach the noise level, namely $728.3-729.125 \mathrm{~cm}^{-1}, \quad 741.975-742.250 \mathrm{~cm}^{-1}, \quad 791.375-$ $792.875 \mathrm{~cm}^{-1}, 763.375-766.375 \mathrm{~cm}^{-1}$. The analysis consisted of the comparison of the averaged residuals with the expected line mixing error spectra. A clear correlation between averaged residuals and line mixing error is noticed, but in general the averaged residuals are smaller than the error spectra indicating that a safe overestimate of the error has been adopted.

In some microwindows the error due to line-mixing is locally comparable with the random error of the single measurement, because the microwindow selection allows this occurrence when positive and negative errors compensate each other.

We conclude that the line-mixing effect is based on a correct model and is correctly taken into account in the microwindow selection.

\subsubsection{Horizontal homogeneity assumption}

The atmosphere sounded by MIPAS is assumed to be horizontally homogeneous. The extension of the atmospheric region observed with limb sounding geometry can reach a length approaching $2000 \mathrm{~km}$. As a consequence, the homogeneity assumption can be critical in presence of pronounced horizontal structures in the atmosphere, as encountered while crossing either the terminator for photochemically active species or the polar vortex in ozone-hole conditions.

Simulations have shown that gradients in temperature are the largest source of error among all gradients (Carli et al., 1998). In Figs. 4 and 5 a standard estimate of a gradient of $1 \mathrm{~K} / 100 \mathrm{~km}$ is made.

In order to verify the impact of the homogeneity assumption, ORM results were compared with those obtained by the GEOFIT retrieval algorithm (Carlotti et al., 2001) that performs the simultaneous retrieval of all the observations acquired along a full orbit and accounts for the horizontal inhomogeneities.

Differences between the results of the two retrieval codes may be larger than the random error bars, but they are not correlated with the horizontal variability of the atmosphere. Therefore the observed differences are to be attributed to the differences between the two models rather than the horizontal homogeneity assumption in the ORM and it was not 
possible to provide a stringent experimental constraint to the amplitude of this error. However, the results of original simulations (Carli et al., 1998) and the absence of a positive detection of any effect suggest that retrieval errors due to horizontal inhomogeneity are estimated with conservative values.

\subsubsection{Local Thermodynamic Equilibrium assumption}

The inclusion of non-LTE emission effects into an operational retrieval algorithm has a high cost in computation time and requires a major effort for modelling and coding all nonLTE processes. However, MIPAS measurements are mostly concentrated in the stratosphere, where the non-LTE effects are generally weak. Furthermore, MIPAS has a high spectral resolution and a wide spectral coverage which enables the selection of ro-vibrational lines of the bands less affected by non-LTE. For these reasons non-LTE modelling was not included in the operational retrieval algorithm and the microwindows selection is made taking into account the nonLTE errors. The non-LTE errors are estimated from pretabulated error propagation matrices which reflect the linear response of the retrieval to perturbations calculated from the difference of Non-LTE and LTE synthetic spectra. Several models are used for the computation of the non-LTE populations of all the relevant species (including also gases not being retrieved operationally but potentially contaminant, such as $\mathrm{CO}$ and NO). A detailed description of the non-LTE models and the species and emitting levels for which non-LTE populations are computed can be found in von Clarmann et al. (1998) and in a subsequent update by López-Puertas et al. (2002).

Ideally, the assessment of non-LTE effects would be best performed by comparing retrieved profiles with co-located accurate and independent measurements taken by instruments not affected by non-LTE. Such measurements were not available in the case of MIPAS pressure and temperature. However, the good agreement found between the temperatures retrieved from the SABER $15 \mu \mathrm{m}$ emission measurements, using a non-LTE model for the $\mathrm{CO}_{2}$ bands very similar to that used in the microwindow selection mentioned above, with rocket in situ measurements and with ground-based lidar measurements (Garcia-Comas et al., 2003; Mertens et al., 2006 , Kutepov et al., 2006) gives us confidence in the $\mathrm{CO}_{2}$ non-LTE model. Non-LTE retrievals, performed from MIPAS data using the operational microwindows in the $15 \mu \mathrm{m}$ fundamental bands and the nonLTE model mentioned above, have shown that non-LTE effects in temperature are smaller than $0.5 \mathrm{~K}$ below $60 \mathrm{~km}$ for all conditions, except in the lower mesosphere when it is sig-

\footnotetext{
${ }^{5}$ Mertens, C. J., Russell III, J. M., Mlynczak, M. G., She, C.-Y., Schmidlin, F. J., Goldberg, R. A., López-Puertas, M., Wintersteiner, P. P., Picard, R. H., Winick, J. R., and Gordley, L. L.: Retrieval of kinetic temperature and carbon dioxide from broadband infrared limb emission measurements taken from the TIMED/SABER instrument, Adv. Space Res., submitted, 2006.
}

nificantly warm, e.g., polar winter or stratospheric warming. In these cases LTE retrievals may underestimate the temperatures by 1 to $3 \mathrm{~K}$ in the $50-70 \mathrm{~km}$ region. In daytime conditions, temperature could be overestimated by 1 to $2 \mathrm{~K}$ at altitudes from 60 to $70 \mathrm{~km}$.

Concerning $\mathrm{O}_{3}$, non-LTE retrievals of $\mathrm{O}_{3}$ from the MIPAS upper atmosphere data in the altitude range from 20 to $100 \mathrm{~km}$ have been compared with "non-LTE free" stellar occultation GOMOS measurements (Verronen et al., 2005) and an agreement within $10-15 \%$ is obtained in the stratosphere and lower mesosphere. These MIPAS retrievals use the same emitting lines from the $14.8 \mu \mathrm{m}$ and $10 \mu \mathrm{m}$ fundamental bands as the operational retrieval, and the same non-LTE model that is used in the microwindow selection. Non-LTE retrievals performed using these microwindows show that non-LTE effects in operational $\mathrm{O}_{3}$ are negligible in the whole altitude range and conditions, except above $55 \mathrm{~km}$ in the polar winter-like conditions (warm lower mesosphere), where ozone can be underestimated by $10-15 \%$ (López-Puertas et al., 2003).

The evaluation of non-LTE effects in the retrieval of water vapour is complicated by non-linear radiative transfer due to saturation effects in the fundamental $(010 \rightarrow 000)$ band used for the retrieval in the upper stratosphere. Non-LTE model errors in retrieved $\mathrm{H}_{2} \mathrm{O}$ profiles can thus have different sign and altitude dependence than in the linear case. A recent study $^{6}$ shows that although vibrational temperatures of the 010 state depart from LTE generally above $60 \mathrm{~km}$, mesospheric non-LTE emissions can enhance measured daytime radiances up to $10 \%$ at tangent heights around $50 \mathrm{~km}$ and $20 \%$ at $60 \mathrm{~km}$ due to solar excitation. The same study demonstrates that the collisional rates used in the $\mathrm{H}_{2} \mathrm{O}$ non-LTE modelling for the MIPAS non-LTE error assessment reproduce the non-LTE effects measured by MIPAS itself.

The estimated non-LTE model error for $\mathrm{H}_{2} \mathrm{O}$ retrieval varies from less than 5\% during night below $50 \mathrm{~km}$ to $30 \%$ at $60 \mathrm{~km}$ during day time. Non-LTE-induced retrieval error of chemically stable species like $\mathrm{H}_{2} \mathrm{O}$ can be assessed by comparing day and night profiles retrieved by similar air masses (i.e. with comparable abundances). Since these species show compact correlations with potential vorticity over a wide altitude range, this assessment can be performed in terms of day and night mean VMR differences at same equivalent latitude (Nash et al., 1996) calculated from ECMWF potential vorticities at given potential temperature levels. Such a comparison of retrieved day and nightime $\mathrm{H}_{2} \mathrm{O}$ (see Fig. 7) reveals systematic daytime enhancements of $5-10 \%$ in the 2000 $2700 \mathrm{~K}(50-60 \mathrm{~km})$ region while above, $\mathrm{H}_{2} \mathrm{O}$ is up to $30 \%$ lower at daytime than at nighttime. These day/night differences can be attributed to non-LTE retrieval errors as it has been demonstrated by retrieving $\mathrm{H}_{2} \mathrm{O}$ under assumption of LTE conditions from simulated spectra (von Clarmann et al.,

\footnotetext{
${ }^{6} \mathrm{M}$. Koukouli, private communication
} 

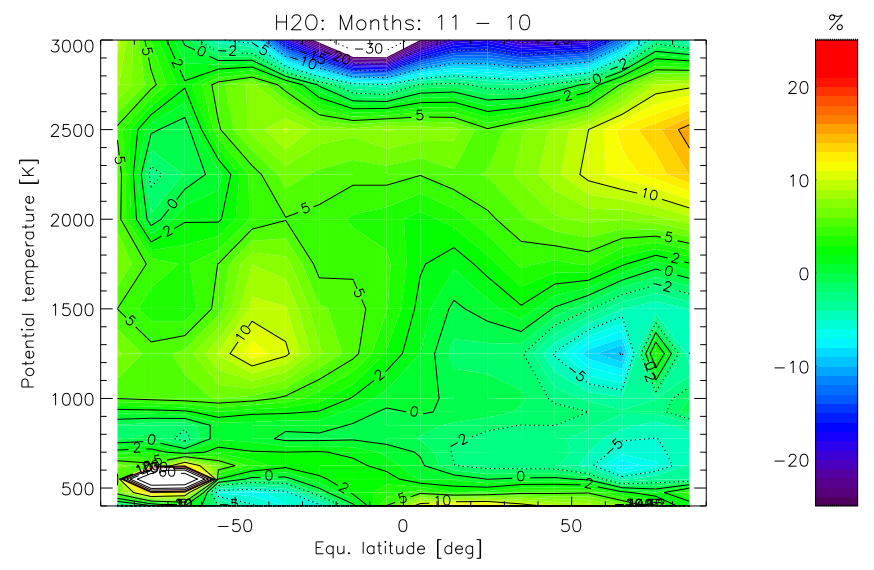

Fig. 7. Day-Night differences in retrieved $\mathrm{H}_{2} \mathrm{O}$ on equivalent latitudes versus potential temperature. The range $500-3000 \mathrm{~K}$ of potential temperature approximately corresponds to the altitude range 20-120 km. Differences represent mean values over the whole observational period (July 2002-March 2003).

2003a). In any case, these non-LTE errors are within the limits of the estimated error budget.

Experimental evidence for non-LTE effects in $\mathrm{CH}_{4}$ emissions in the $v_{4}$ band was found by López Puertas et al. (2005). Non-LTE minus LTE limb radiance differences have been detected above $45 \mathrm{~km}$, increasing up to $20 \%$ at $60 \mathrm{~km}$. This study demonstrated that the non-LTE model used for the non-LTE model error assessment of MIPAS $\mathrm{CH}_{4}$ fully reproduces the observed non-LTE features. The estimated nonLTE model errors are less than $2 \%$ below $50 \mathrm{~km}$ and larger than $20 \%$ at $60 \mathrm{~km}$. These values are also in consonance with the day/night differences of three month means of retrieved $\mathrm{CH}_{4}$ (see Fig. 8). Higher daytime $\mathrm{CH}_{4}(5-10 \%)$ is retrieved above about $45 \mathrm{~km}$ (potential temperature of $1700 \mathrm{~K}$ ) under LTE assumption due to population enhancements by solar excitation.

Possible non-LTE effects in the retrieval of $\mathrm{N}_{2} \mathrm{O}$ have been estimated on the basis of theoretical non-LTE population calculations which indicate non-LTE-induced deviations of less than $1 \%$ for retrieved $\mathrm{N}_{2} \mathrm{O}$. No evidence of non-LTE effects in $\mathrm{N}_{2} \mathrm{O}$ emissions has been found so far. Also, day/night differences of 3 month mean retrieved $\mathrm{N}_{2} \mathrm{O}$ (see Fig. 9) do not hint at systematic deviations due to non-LTE neglect in the retrieval.

Non-LTE effects in the $6.2 \mu \mathrm{m}$ region used for the retrieval of $\mathrm{NO}_{2}$ have been analysed in detail by Funke et al. (2005). Non-LTE effects in retrieved $\mathrm{NO}_{2}$ cannot be evaluated by means of day/night differences due to diurnal variations of this key species.

The chemical excitation rate of the $\mathrm{NO}_{2} v_{3}$ states emitting in this spectral region was found to be 50 times less than estimated in previous studies. In the study of Funke et al. (2005), the non-LTE retrieval of $\mathrm{NO}_{2}$ was performed using the up-
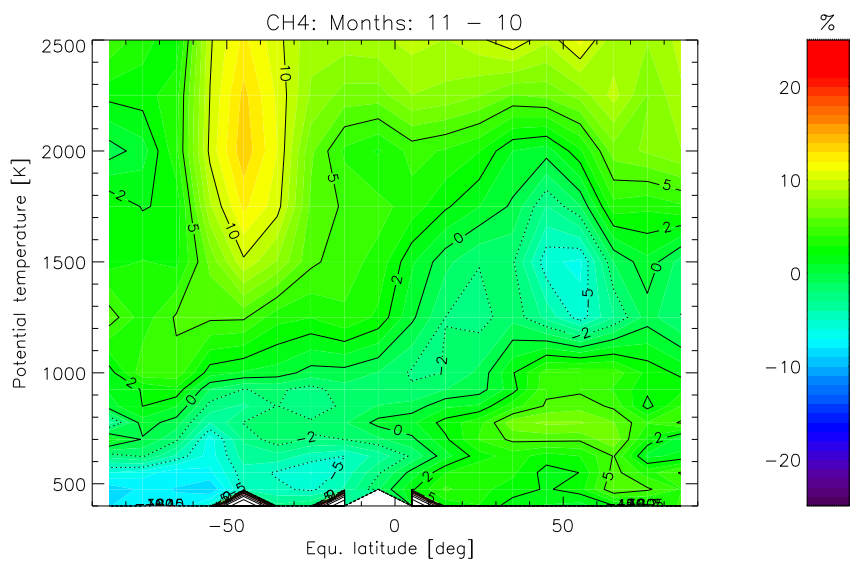

Fig. 8. Day-Night differences in retrieved $\mathrm{CH}_{4}$ on equivalent latitudes versus potential temperature. Differences represent mean values over the whole observational period (July 2002-March 2003).

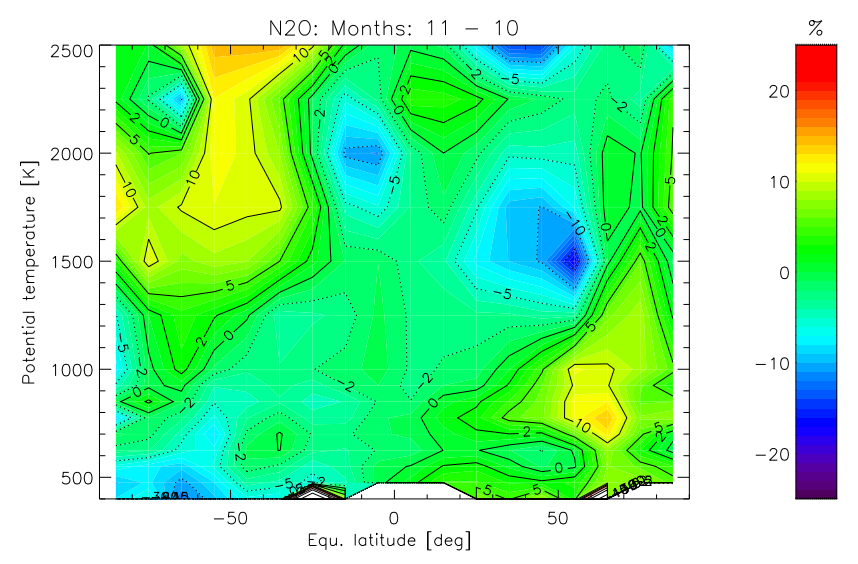

Fig. 9. Day-Night differences in retrieved $\mathrm{N}_{2} \mathrm{O}$ on equivalent latitudes versus potential temperature. Differences represent mean values over the whole observational period (July 2002-March 2003).

dated rates and results were found to be in good agreement with non-LTE-free HALOE data. These new rates have also been incorporated in the assessment of non-LTE errors in the ORM retrieval of $\mathrm{NO}_{2}$. No significant error is expected below $50 \mathrm{~km}$. Above, the LTE assumption leads to an underestimation of $\mathrm{NO}_{2}$ by $10-50 \%$ with highest deviations at polar winter conditions.

\section{Computation of the total error of retrieved profiles}

According to Eq. (5), the total error is obtained as a quadratic sum of measurement and forward model errors. The different components of this budget can be acquired from different sources.

The measurement error $\mathbf{V}_{x}$ is computed by level 2 processor and is reported in the level 2 output files. 


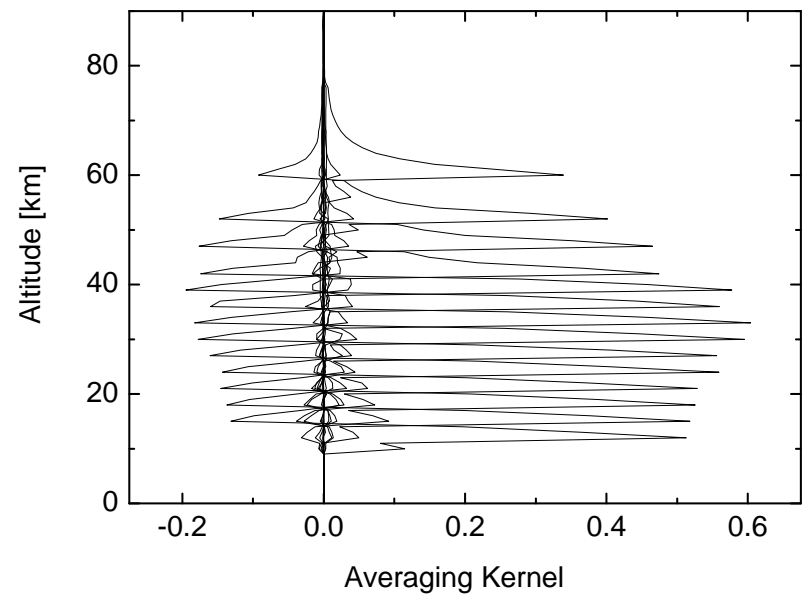

Fig. 10. Averaging kernels for the ozone retrieval in the case of $20^{\circ} \mathrm{N}-65^{\circ} \mathrm{N}$ latitude band in July.

The forward model errors $\mathbf{V}_{x}^{i}$, of which only the diagonal elements are provided, are available at http://www-atm. physics.ox.ac.uk/group/mipas/err. In this database, estimates are given for the five standard atmospheres.

It has to be noticed that the error in VMR profiles due to the propagation of $\mathrm{p}$ and $\mathrm{T}$ errors, which is reported in the above data base, does not include the effect of forward model errors in $\mathrm{p}$ and $\mathrm{T}$ retrieval and does not account for the variability of the $p, T$ retrieval errors. For a more accurate estimate of the $\mathrm{p}, \mathrm{T}$ error propagation it is necessary to propagate, according to a suitable propagation matrix $\mathbf{E}$ as described in Sect. 4.2.4, the total error budget of the individual $\mathrm{p}$, T retrievals into the VMR retrieval.

The propagation matrices $\mathbf{E}$ for the different species are included in the level 2 auxiliary data set and are available at http://www.ifac.cnr.it/retrieval/auxiliary.html.

Access to the individual contribution allows, when necessary, to separate the random and systematic components of the error budget. Indeed, most of the forward model errors can be either random or systematic according to the ensemble of measurements that is being considered. For example, errors such as the propagation of the random pT error into VMR retrievals will be "systematic" if considering, for example, the sum of $2 * \mathrm{CH}_{4}+\mathrm{H}_{2} \mathrm{O}$ at one altitude within one scan since both the $\mathrm{CH}_{4}$ and $\mathrm{H}_{2} \mathrm{O}$ retrievals share the same error in temperature retrieval for that altitude. However, when comparing $\mathrm{CH}_{4}$ retrievals for 2 successive scans, the contributions of the temperature errors will be uncorrelated. So the $\mathrm{p}, \mathrm{T}$ error can be regarded as random for time/length scales beyond $1 \mathrm{scan}(75 \mathrm{~s} / 500 \mathrm{~km})$. Contaminating species, such as CFC-11, are modelled assuming a zonal mean climatology. The actual atmospheric CFC-11 distribution is likely to depart from the climatology in a correlated manner with, say, a 30 degree latitude bin on a particular day but not within the same latitude bin when combining data from different months. So this error can be regarded as random for time/length scales beyond 1 month $/ 3000 \mathrm{~km}$.

\section{Averaging kernels}

For a full characterization of the retrieved profiles, along with the error information it is necessary to know how the description of the true state of the atmosphere is affected by the observing system. This information is provided by the averaging kernel matrix (AKM) and is indispensable for the validation operations when comparison between different measurements with different vertical resolution of the same atmospheric vertical profiles are performed (Ceccherini et al., 2003; Rodgers and Connor, 2003; Ridolfi et al., 2006). It is also required for comparisons to models with higher vertical resolution than MIPAS.

In the linear approximation, the relation connecting the retrieved profile with the true state of the atmosphere is given by:

$\hat{\boldsymbol{x}}-\hat{\boldsymbol{x}}_{0}=\mathbf{A}\left(\boldsymbol{x}-\boldsymbol{x}_{0}\right)$,

where $\hat{x}$ is the retrieved profile when the true state of the atmosphere is $\boldsymbol{x}, \hat{\boldsymbol{x}}_{0}$ is the retrieved profile when the true state of the atmosphere is the linearization state $\boldsymbol{x}_{0}$ and $\mathbf{A}=\left.\frac{\partial \hat{\boldsymbol{x}}}{\partial \boldsymbol{x}}\right|_{\boldsymbol{x}_{0}}$ is the Averaging Kernel Matrix.

The rows and the columns of $\mathbf{A}$ are respectively the averaging kernels (AK) and the delta-function responses. There is one AK for each retrieved quantity and one delta function for each grid point used to represent the AKs. The AKs are in general functions peaking at the grid point that coincides with the altitude of the retrieved quantity and have a width that is a measurement of the vertical resolution of the retrieved parameter. When the grid used for the representation of the AKs coincides with the altitudes of the retrieved quantities (measurement grid) and no a-priori information is used for the retrieval, $\mathbf{A}$ is the identity matrix.

In the case of MIPAS no a priori information is used and $\mathbf{A}$ differs from the identity matrix only when a fine grid is used for the representation of the AKs. The fine grid is chosen to have points that coincide with the measurement grid as well as extra intermediate points.

The AKs are calculated on a fine grid of $1 \mathrm{~km}$ for both NRT and OL measurements using the climatological profile $\boldsymbol{x}_{0}$ as linearization state. The AKs, as well as the climatological profiles, are available on the web at http://www.ifac.cnr.it/ retrieval/auxiliary.html.

The AKs are given as a function of altitude. From the atmospheric status it is possible to derive the corresponding values of pressure for which the AKs are calculated. However, this is the pressure grid of a nominal measurement and is in general different from that of a real measurement.

For small differences in the measurement grid, the shape of the AK depends more on the relative location of the measurement points than on their actual altitude. Therefore, the 

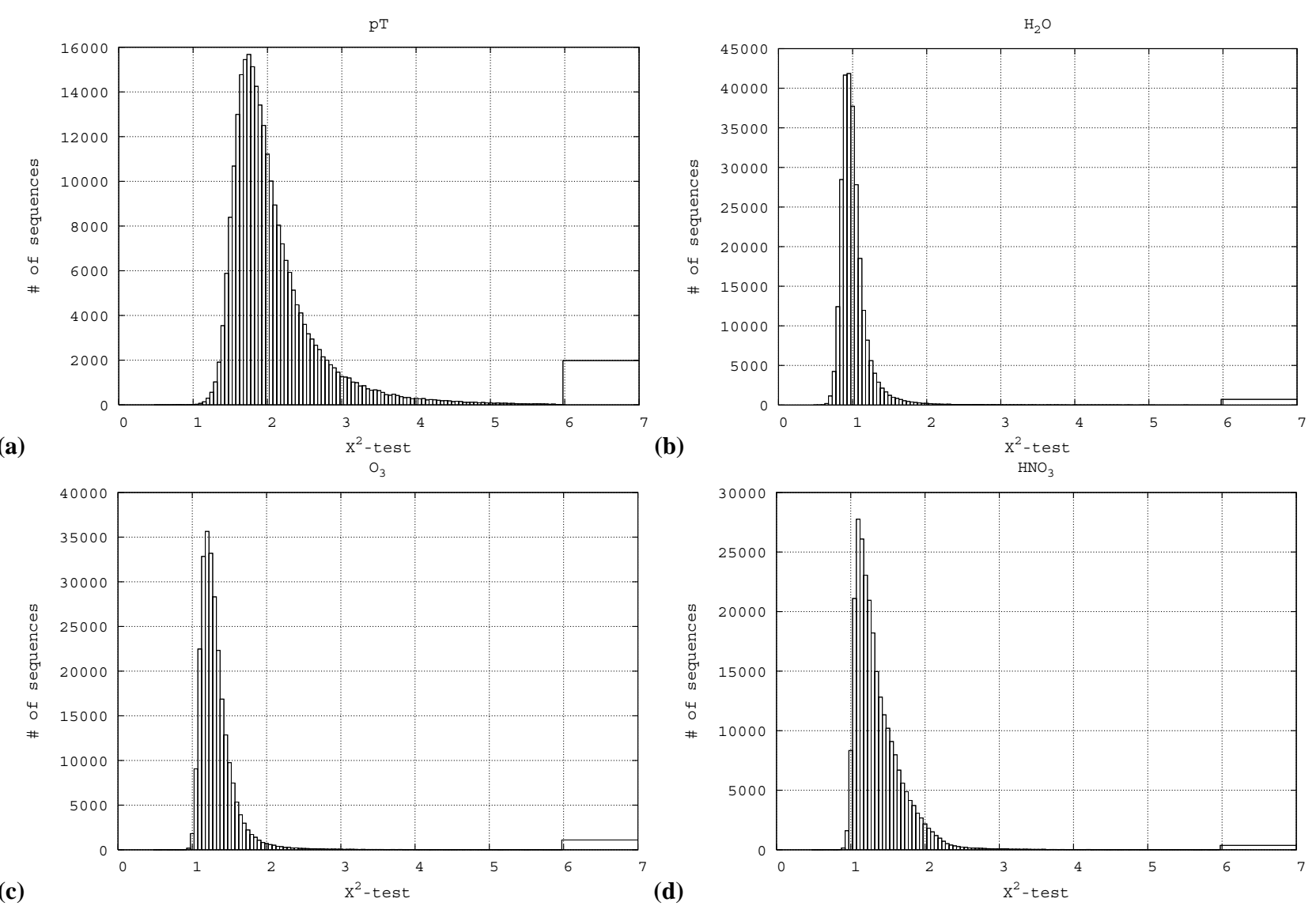

Fig. 11. Histograms of $\chi^{2}$ test distribution for $\mathrm{p}, \mathrm{T}, \mathrm{H}_{2} \mathrm{O}, \mathrm{O}_{3}$ and $\mathrm{HNO}_{3}$ retrievals. In each histogram, a constant box width is used for events with $\chi^{2}$ values not larger than 6 , while the last box on the right counts all the events with $\chi^{2}$ larger than 6 .

AKs calculated for a nominal measurement grid can be used to define the AKs of the real measurement grid. Namely, the measured tangent pressure can be associated with the values of the AKs at the measurement grid. This implies a stretching/shrinking of the representation grid and an interpolation is needed for the determination of the pressure of the intermediate points. Assuming a logarithmic variation of pressure with altitude, if $\boldsymbol{p}_{\text {nom }}$ and $\boldsymbol{p}_{r}$ are the pressure of the nominal and real pressure grid respectively, the pressure of the intermediate point can be determined from the following expression:

$\ln \boldsymbol{p}_{r}(i+j)=\ln \boldsymbol{p}_{r}(i)+\frac{\left(\ln \frac{\boldsymbol{p}_{r}(i+n)}{\boldsymbol{p}_{r}(i)}\right)}{\left(\ln \frac{\boldsymbol{p}_{\mathrm{nom}}(i+n)}{\boldsymbol{p}_{\mathrm{nom}}(i)}\right)} \ln \frac{\boldsymbol{p}_{\text {nom }}(i+j)}{\boldsymbol{p}_{\text {nom }}(i)}$,

where $i$ is the index of a measurement grid point, $(n-1)$ is the number of intermediate points, $i+n$ is the index of the next measurement grid point and $j=1, n-1$ is the index of the intermediate grid point.

Outside the retrieval range:

$\boldsymbol{p}_{r}(l)=\boldsymbol{p}_{\text {nom }}(l) \frac{\boldsymbol{p}_{r}(k)}{\boldsymbol{p}_{\text {nom }}(k)}$, where $l$ is the index of the extrapolated point and $\boldsymbol{p}_{r}(\mathrm{k})$ and $\boldsymbol{p}_{\text {nom }}(\mathrm{k})$ are the pressure of the highest/lowest retrieved point in the real and nominal grid, respectively.

This process provides the averaging kernel on an irregular pressure grid that corresponds to the specific limb measurements.

The AKs in the case of ozone retrieval for the $20^{\circ} \mathrm{N}-65^{\circ} \mathrm{N}$ latitude band in July represented on a grid of $1 \mathrm{~km}$ step are shown in Fig. 10.

The AK cannot always be defined. When the AKM is calculated using different "starting numbers" for the generation of the random errors added to the spectra the same AKMs should be obtained. If different AKMs are obtained it means that the measurement error can change the retrieved values of a large amount and the retrieval of that parameter is illconditioned. In these cases, occurring for some of the additional altitudes available in the OL products, the averaging kernels are not provided, indicating that the corresponding retrieved value has no physical meaning and can only be used to characterise the retrieval process from the mathematical point of view (see Sect. 3.3). 

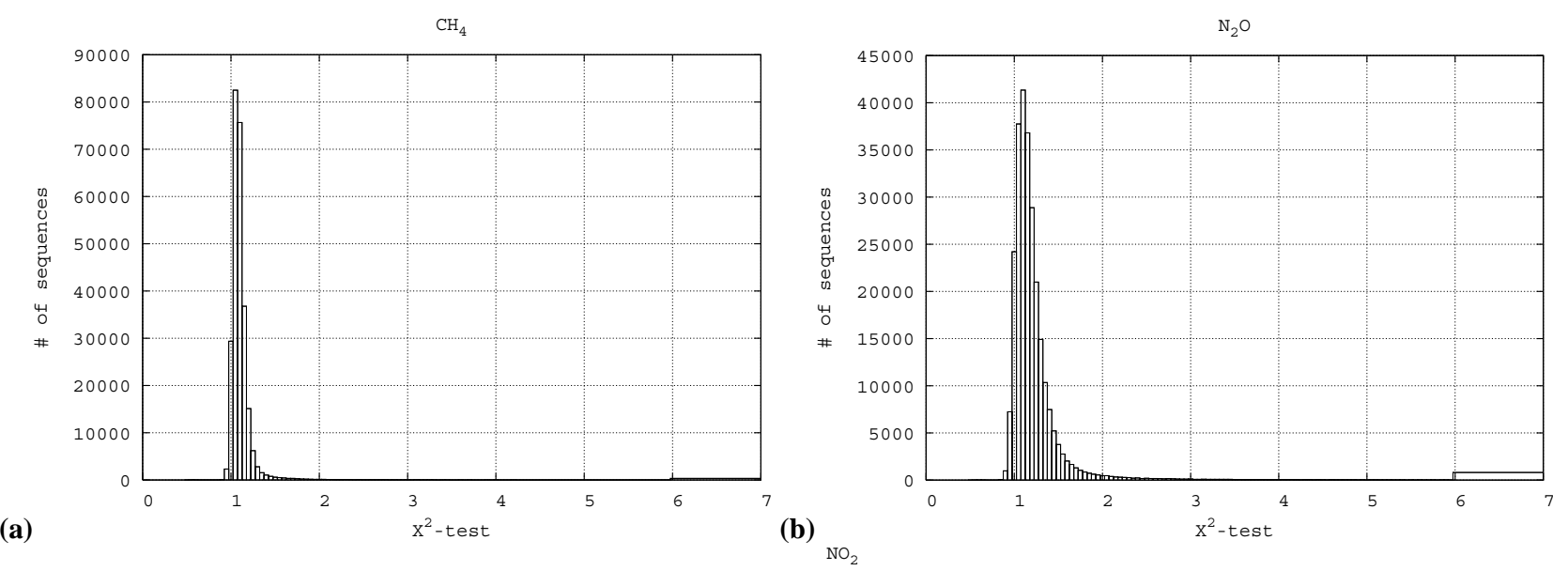

(a)

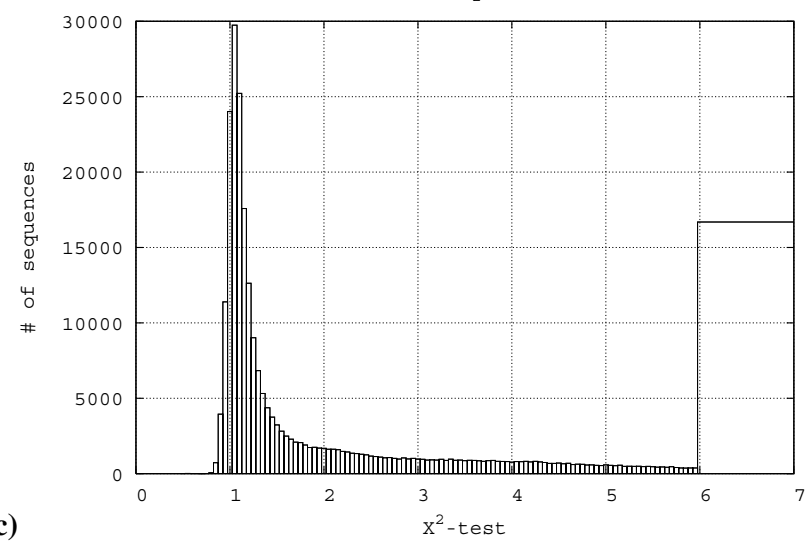

(c)

Fig. 12. As Fig. 11, showing histograms of $\chi^{2}$ test distribution for $\mathrm{CH}_{4}, \mathrm{~N}_{2} \mathrm{O}$ and $\mathrm{NO}_{2}$ retrievals.

Table 4. Statistics of ORM performances.

\begin{tabular}{lccccccc}
\hline & $\mathrm{PT}$ & $\mathrm{H}_{2} \mathrm{O}$ & $\mathrm{O}_{3}$ & $\mathrm{HNO}_{3}$ & $\mathrm{CH}_{4}$ & $\mathrm{~N}_{2} \mathrm{O}$ & $\mathrm{NO}_{2}$ \\
\hline Measured mean $\chi^{2}$-test & 2.09 & 1.03 & 1.34 & 1.37 & 1.12 & 1.25 & 2.22 \\
Estimated $\chi^{2}$-test & 2.82 & 1.76 & 1.79 & 1.11 & 2.23 & 1.27 & 1.23 \\
$\begin{array}{l}\text { Measured standard deviation } \\
\text { Statistical standard deviation } \\
\text { (due to the finite number of }\end{array}$ & 0.72 & 0.36 & 0.44 & 0.39 & 0.30 & 0.52 & 2.03 \\
$\begin{array}{l}\text { average residuals only) } \\
\text { \% of successful retrievals }\end{array}$ & 9.035 & 0.03 & 0.03 & 0.03 & 0.035 & 0.03 \\
& & & & & & & \\
\hline
\end{tabular}

\section{ORM performances}

The $\chi^{2}$-test of each retrieval, given by the value of $\chi^{2}$ function (see Eq. 1) at convergence divided by the Number of Degrees of Freedom (NDF) can be used as a diagnostic tool for the evaluation of the code performances.

All data measured in the year 2003 that were reprocessed by the ESA OL processor have been used to evaluate the mean $\chi^{2}$-test value of each orbit. The histograms of the mean $\chi^{2}$ values for the different species are shown in Fig. 11 and
Fig. 12. In each histogram, a constant box width is used for events with $\chi^{2}$ values not larger than 6 , while the last box on the right counts all the events with $\chi^{2}$ larger than 6 .

With the only exception of $\mathrm{NO}_{2}$, that shows occasional large values which have been proved to be due to an error in Level 2 processor, the $\chi^{2}$ of the other species are distributed around a constant average value.

Table 4 shows for all target species the one-year mean of the measured $\chi^{2}$ test. Deviations of the $\chi^{2}$ test from unity 

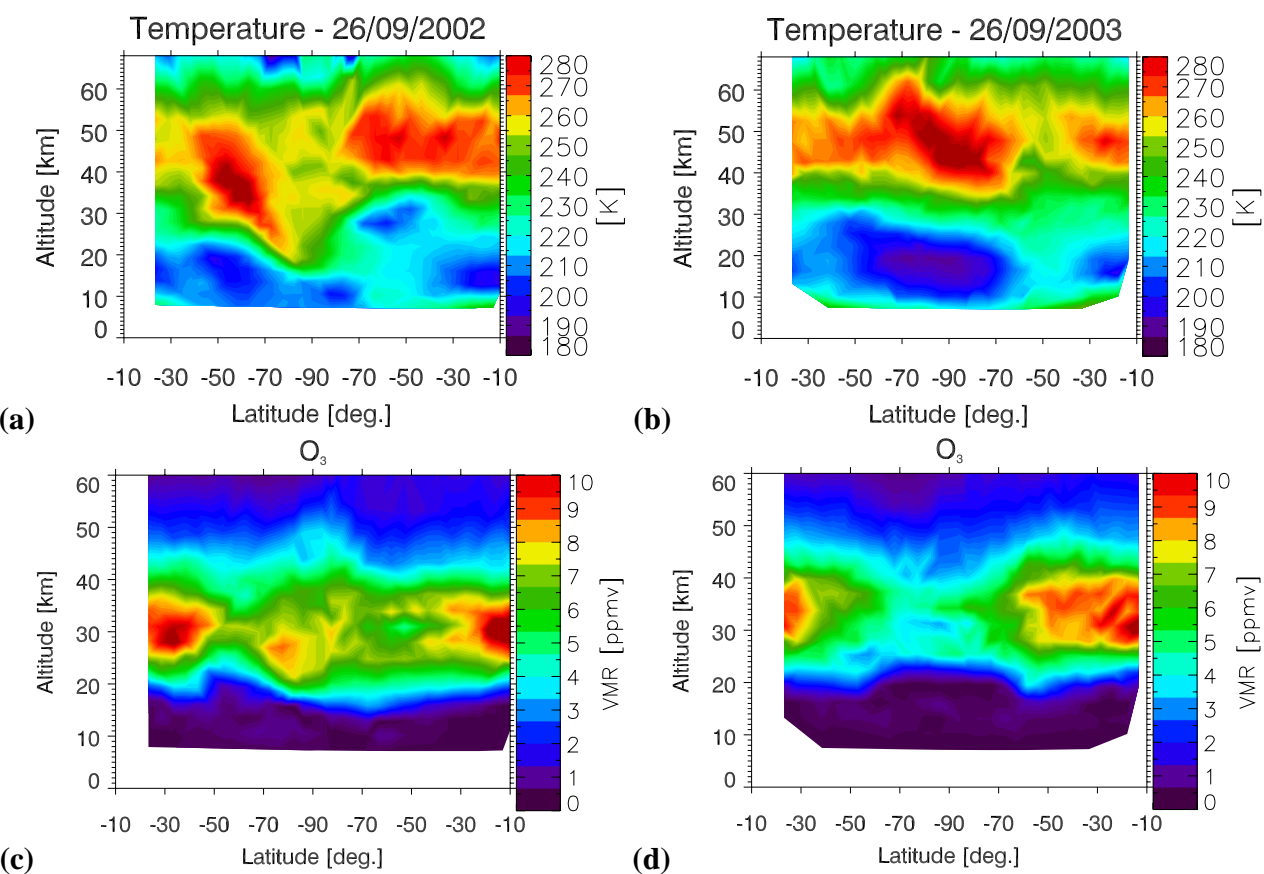

Fig. 13. Maps of temperature and VMR profiles of $\mathrm{O}_{3}$ located around South Pole obtained from the analysis of two orbits measured on 26 September 2002 (maps on the left) and 26 September 2003 (maps on the right), respectively.

provide an indication of the existence of forward model errors even if not all forward model errors do equally manifest themselves through this test, due to possible correlations among errors and between errors and the target species. The estimated $\chi^{2}$ test, calculated from the a priori estimates of forward model and random errors, is also reported in the table. In the limit in which the amplitude of the residuals can be assumed to be a measurement of the amplitude of the retrieval error, a $\chi^{2}$-test equal to 2 implies that the amplitude of the forward model errors is equal to the amplitude of the random errors.

In general we find that the $\chi^{2}$ test varies from 1 to 2 and that a good agreement is obtained between measured and estimated values. For most retrievals, the measured $\chi^{2}$ are smaller than the calculated ones (notably a reduction of a factor 2 is observed in the case of $\mathrm{CH}_{4}$ ) confirming that most probably conservative estimates of the forward model errors are currently made, the only exception being the already mentioned case of $\mathrm{NO}_{2}$. Nevertheless, this test does not provide the information of which error and by how much may be overestimated.

The third row of the table shows the standard deviation of the $\chi^{2}$ statistics as measured from the one-year distribution. The measured standard deviation is the results of three main contributions: statistical standard deviation, due to the finite number of averaged residuals and equal to $\sqrt{\frac{2}{\mathrm{NDF}}}$, spread in the statistics of the $\chi^{2}$ test due to forward model errors with variable amplitude and spread associated with convergence criteria. The statistical standard deviation, also reported in the 4th row, is always very small and has a negligible effect.

It has been verified that the spread due to convergence criteria provides the main contribution to the measured standard deviation for all retrievals except $p, T$ and $\mathrm{H}_{2} \mathrm{O}$ retrievals. For these two retrievals, the presence of forward model errors with variable amplitude has to be considered as the main contribution to the measured standard deviation.

The last line of Table 4 shows that a good percentage of retrievals have successfully reached convergence.

Concerning the run-time performances, the computing time needed to process a complete orbit on a linux machine with 2 processors Intel Xeon of $2.8 \mathrm{GHz}$ ( 4 Gbyte RAM) is about $43 \mathrm{~min}$. for NRT analysis and about $80 \mathrm{~min}$. for OL analysis. Considering that the measuring time of one orbit is about $100 \mathrm{~min}$, the objective of NRT processing has been met in both cases.

\section{Example of the results}

The polar orbit of the ENVISAT satellite provides a nearly full coverage of the globe in one day. The global coverage, combined with the MIPAS capability of retrieving, simultaneously and during both day and night, the vertical profile of numerous species, allows continuous measurement of the three dimensional (versus latitude, longitude and altitude) 

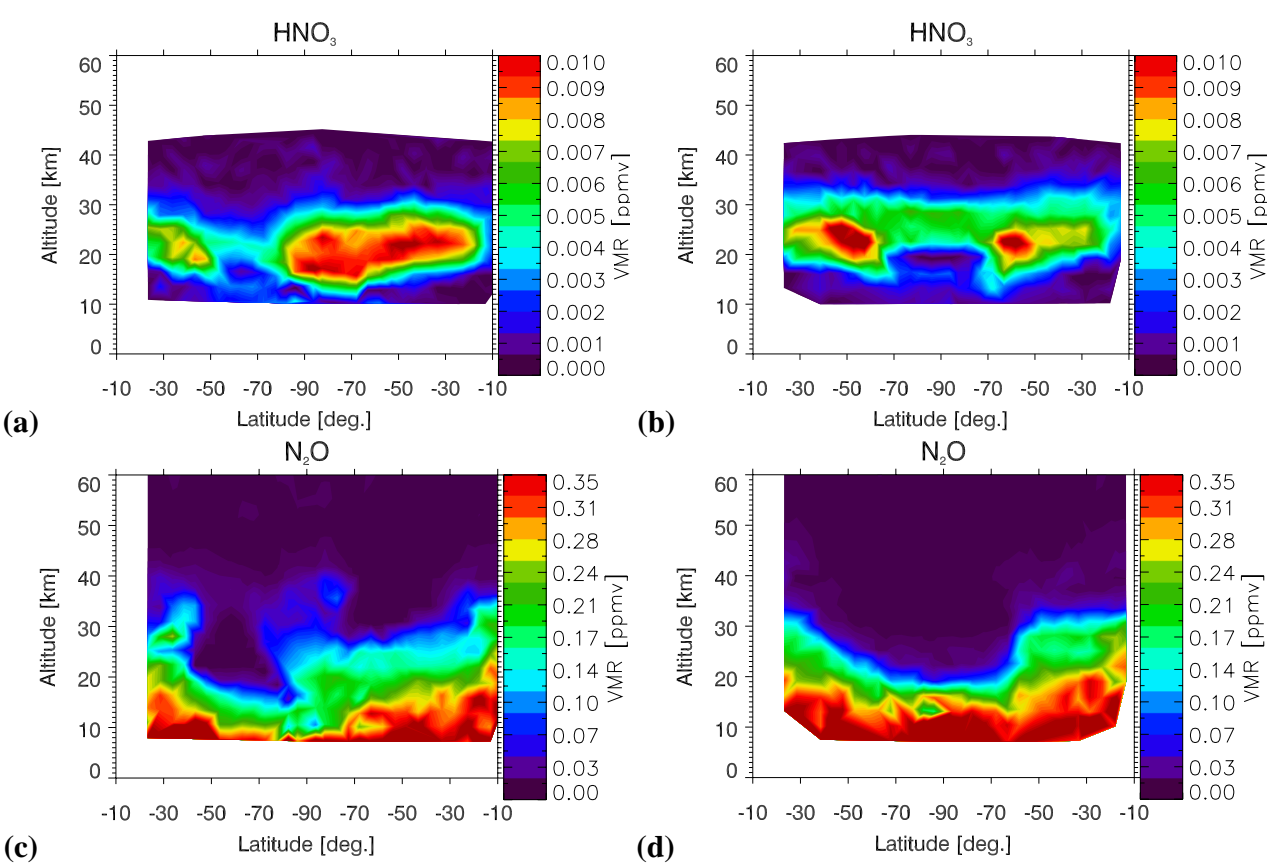

Fig. 14. As Fig. 13, showing maps of $\mathrm{VMR}$ profiles of $\mathrm{HNO}_{3}$ and $\mathrm{N}_{2} \mathrm{O}$ obtained from the analysis of two orbits measured on 26 September 2002 (maps on the left) and 26 September 2003 (maps on the right), respectively.

atmospheric composition as a function of time. This capability represents a major achievement with respect to previous measurements.

The fact that not only a key constituent is measured, but a set of interlinked species are simultaneously determined is of particular importance from the scientific point of view. As an example of this potentiality of MIPAS measurements a qualitative comparison is shown between the Antarctic ozone hole in 2002 and 2003.

The maps of $\mathrm{T}, \mathrm{O}_{3}, \mathrm{~N}_{2} \mathrm{O}$ and $\mathrm{HNO}_{3}$ obtained by ORM from the analysis of a single orbit on 26 September in the two years are shown in Figs. 13 and 14.

The maps relative to the year 2002 highlight a strongly perturbed Antarctic stratospheric vortex, due to a major warming which occurred very early that year, while in 2003 at the same date the vortex still appears very stable (as inferred by the temperature map) and with a subsidence extended over a large area (as inferred by the $\mathrm{N}_{2} \mathrm{O}$ map). The minim in $\mathrm{HNO}_{3}$ concentration is a well-known climatological feature of the Antarctic vortex at this time of the year (September) which has been shown (Crutzen and Arnold, 1986) to be due to denitrification, i.e. irreversible removal of nitrogen to lower levels through sedimentation of polar stratospheric clouds. $\mathrm{O}_{3}$ depletion correlates with denitrification at low altitudes (chemical depletion) and with subsidence at high altitudes (dynamical depletion). The different dynamics and chemistry observed in the two years lead to a significantly different pattern in ozone distribution. The vertically resolved measurements of MIPAS easily highlight the on-going pro- cesses and resolve the two regimes of behaviour in the two years.

The geographical distribution of the chemical depletion can be observed in vertically resolved stereographic maps as shown in Fig. 15 where $\mathrm{O}_{3}$ and $\mathrm{HNO}_{3}$ are plotted for the two years at an altitude of $21 \mathrm{~km}$. The MIPAS observations made during the ozone hole break-up in 2002 are further described in a number of papers, mostly collected on a special issue on Antarctic vortex $2002^{7}$.

\section{Conclusions}

The performances of the level 2 analysis of MIPAS instrument during the first two years of operations have been reviewed. The features of the ORM code, which is the basis of the operational level 2 analysis, have been recalled and the approximations and assumptions, implemented in the code in order to meet the NRT and accuracy requirements, have been critically reviewed testing their impact on the retrieved profiles in the case of real measurements.

The retrieval code has successfully processed, in NRT, MIPAS measurements without any change in the level 2 processor. Changes have only been made in the pre-processor with the addition of an algorithm for the rejection of measurements contaminated by clouds (cloud filtering).

A refinement of both the retrieval settings and the spectroscopic database has proved to be important for the quality

\footnotetext{
${ }^{7}$ J. Atmos. Sci., vol. 62, number 3 (March 2005).
} 
(a)

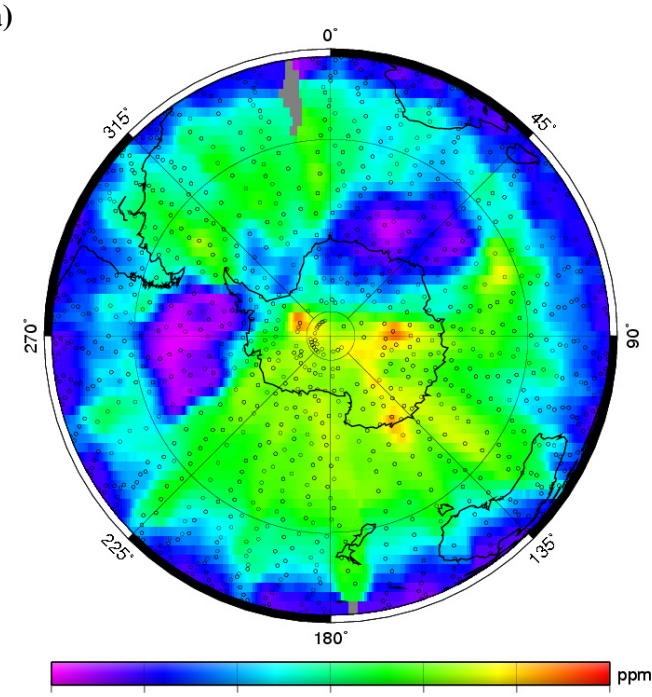

(c)
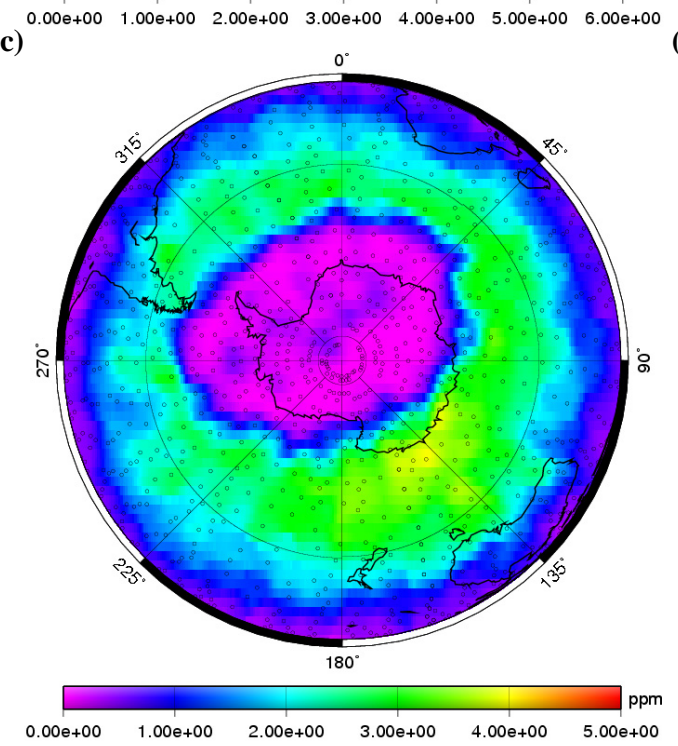

(b)

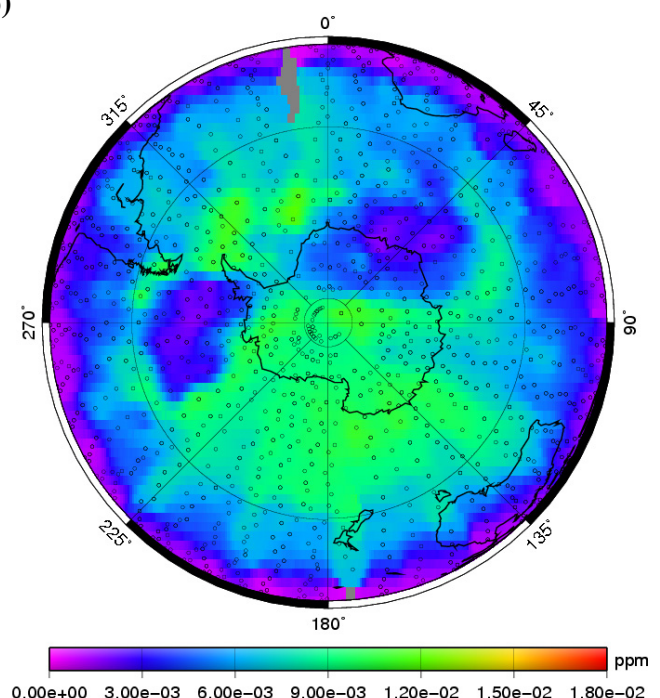

(d)

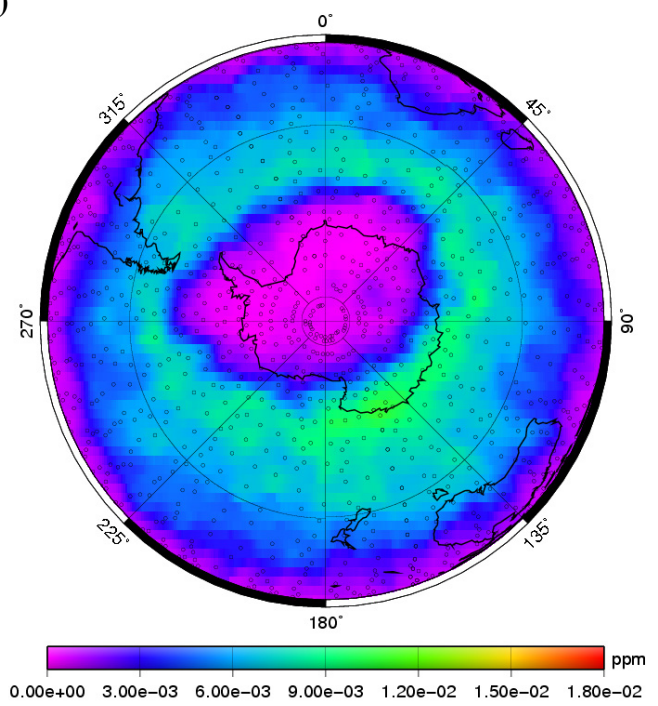

Fig. 15. Polar stereographic maps of $\mathrm{O}_{3}$ (maps on the left) and $\mathrm{HNO}_{3}$ (maps on the right) obtained from 3 days of measurements (25-27 September 2002, upper plots and 25-27 September 2003, lower plots). The dots identify the measurements location.

of the products. In particular, the convergence error can be reduced using more stringent convergence criteria and the extrapolation error, due to the profile assumptions outside the retrieval range, can be reduced using an extended retrieval range. Unfortunately, these error reductions can only be attained at the expenses of computing time and have not been implemented in the NRT analysis. These improvements have been implemented in the OL products, which have a less stringent constraint for the processing time and are produced some time after the measurement using the same code and better geolocated data.

A review of the total error budget of the retrieved profiles was performed on the basis of the results of instrument and level 1 processor characterisation, and of specific tests made on the level 2 data. The error budget and the overall perfor- mances of the level 2 processor are verified with a statistical analysis of the mean $\chi^{2}$-test of the measurements obtained during a whole year of MIPAS operation. We find that in general the chi-square is smaller than our a priori estimate indicating that conservative forward model errors have been assumed. In the error budget the forward model errors are comparable with the random errors as required in an optimized retrieval, however, the total error is larger than the possibilities of the MIPAS instrument. Further work is needed to reduce the amplitude of forward model errors so that the random error can also be reduced with the analysis of more microwindows and a smaller total error is obtained. 
Acknowledgements. This study was supported by ESA contracts $11717 / 95 / \mathrm{NL} / \mathrm{CN}$ and $17580 / 03 / \mathrm{I}-\mathrm{OL}$. The study team is grateful to H. Nett, J. Langen and R. Koopman for the fruitful discussions and for the efficient coordination of MIPAS NRT code development studies. We thank M. Koukouli and the other anonymous referees for their useful comments.

Edited by: P. Hartogh

\section{References}

Abrams, M. C., Goldman, A., Gunson, M. R., Risland, C. P., and Zander, R.: Observations of the infrared solar spectrum from space by ATMOS experiment, Appl. Opt., 34, 2747-2751, 1996.

Birk, M. and Wagner, G.: Radiometric accuracy assessment of MIPAS on ENVISAT, Proceedings of ENVISAT Calibration Review, Nordwick, ESA-ESTEC, ESA Publication SP-520, 2002.

Carli, B., Ceccherini, S., Raspollini, P., Dinelli, B. M., Dudhia, A., and Echle, G.: Final Report of the Study of the retrieval of atmospheric trace gas profiles from infrared spectra', ESA Contract 12055-96-NL-CN, 1998.

Carli, B., Ceccherini, S., Gignoli, A., et al.: Final Report of the Study 'Development of an optimised algorithm for routine $\mathrm{p}$, $\mathrm{T}$ and VMR retrieval from MIPAS limb emission spectra, ESA Contract 11717/95/NL/CN, 2004.

Carlotti, M.: Global-fit approach to the analysis of limb-scanning atmospheric measurements, Appl. Opt., 27, 3250-3254, 1988.

Carlotti, M. and Ridolfi, M.: Derivation of temperature and pressure from submillimetric limb observations, Appl. Opt., 38, 23982409, 1999.

Carlotti, M., Dinelli, B. M., Raspollini, P., and Ridolfi, M.: Geofit approach to the analysis of limb-scanning satellite measurements, Appl. Opt., 41, 1872-1885, 2001.

Ceccherini, S., Carli, B., Pascale, E., Prosperi, M., Raspollini, P., and Dinelli, B. M.: Comparison of measurements made with two different instruments of the same atmospheric vertical profile, Appl. Opt., 42(32), 6465-6473, 2003.

Chipperfield, M. P.: Multiannual Simulations with a ThreeDimensional Chemical Transport Model, J. Geophys. Res., 104, 1781-1805, 1999.

Crutzen, P. J. and Arnold, F.: Nitric acid cloud formation in the cold Antartic stratosphere: a major cause for the springtime 'ozone hole', Nature, 324, 651-655, 1986.

Dudhia, A.: REC analysis of MIPAS data, Technical Note, of ESA Contract no. 11717/95/NL/CN, 2002.

Dudhia, A., Jay, V. L., and Rodgers, C. D.: Microwindow selection for high-spectral-resolution sounders, Appl. Opt., 41, 36653673, 2002a.

Dudhia, A., Morris, P. E., and Wells, R. J.: Fast monochromatic radiative transfer calculations for limb sounding, J. Quant. Spectr. Radiat. Trans., 74, 745-756, 2002 b.

Edwards, P.: High Level algorithm definition document of the MIPAS Reference Forward Model, ESA Report PO-TN-OXF-GS0004, 1997.

Edwards, D. P. and Strow, L. L.: Spectral line shape considerations for limb temperature sounders, J. Geophys. Res., 96, 20859 $20868,1991$.

Fischer, H., Blom, C., Oelhaf, H., Carli, B., Carlotti, M., Delbouille, L., Ehhalt, D., Flaud, J.-M., Isaksen, I., Lopez-Puertas,
M., McElroy, C. T., and Zander, R.: Envisat-MIPAS - An instrument for atmospheric chemistry and climate research, edited by: Readings, C. and Harris, R. A., ESA Publication SP-1229, European Space Agency, 2000.

Flaud, J. M. and Piccolo, C.: Spectroscopic Database Updates, Technical Note of the ESA study no. 11717/95/NL/CN, July 2001.

Flaud, J. M. and Piccolo, C.: MIPAS_03: an update of the MIPAS.PF2 database, September 2003, Technical Note of the ESA study no. 11717/95/NL/CN., 2003.

Flaud, J.-M., Piccolo, C., Carli, B., Perrin, A., Coudert, L. H., Teffo, J.-L., and Brown, L. R.: Molecular line parameters for the MIPAS (Michelson Interferometer for Passive Atmospheric Sounding) experiment, J. Atmos. Ocean Opt., 16, 172-182, $2003 \mathrm{a}$.

Flaud, J.-M., Perrin, A., Orphal, J., Kou, Q., Flaud, P.-M., Dutkiewicz, Z., and Piccolo, C.: New analysis of the u5+u9-u9 hot band of $\mathrm{HNO}_{3}$, J. Quant. Spectr. Radiat. Trans., 77, 355-364, 2003b.

Flaud, J.-M., Wagner, G., Birk, M., Camy-Peyret, C., Claveau, C., De Backer-Barilly, M. R., Barbe, A., and Piccolo, C.: The Ozone absorption around $10 \mathrm{~mm}$, J. Geophys. Res.-Atmos., 108(D9), 4269, doi:10.1029/2002JD002755, 2003c.

Friedl-Valloon, F., Maucher, G., Seefeldner, M., Trieschmann, O., Kleinert, A., Lengel, A., Keim, C., Oelhaf, H., and Fischer, H.: Design and characterisation of the balloon-borne Michelson Interferometer for Passive Atmospheric Sounding (MIPAS-B2), Appl. Opt., 43, 3335-3355, 2004.

Funke, B., López-Puertas, M., Clarmann, v. T., Stiller, G. P., Fischer, H., Glatthor, N., Grabowski, U., Höpfner, M., Kellmann, S., Kiefer, M., Linden, A., Mengistu Tsidu, G., Milz, M., Steck, T., and Wang, D. Y.: Retrieval of stratospheric NO and NO2 from 5.3 and $6.2 \mu \mathrm{m}$ non-LTE emissions measured by MIPAS on ENVISAT, J. Geophys. Res., 110, D09302, doi:10.1029/2004JD005225, 2005.

Garcia-Comas, M., López-Puertas, M., Mertens, C. J., Wintersteiner, P. P., Picard, R. H., Winick, J. R., Mlynczak, M. G., Remsberg, E. E., Russell III, J. M., and Gordley, L. L.: Comparisons of SABER non-LTE retrievals of kinetic temperature with ground-based measurements, Geophys. Res. Abstr., 5, 10148 , 2003.

Greenhough, J., Remedios, J. J., Sembhi, H., and Kramer, L. J.: Towards cloud detection and cloud frequency distributions from MIPAS infra-red observations, Adv. Space Res., 36, 800-806, 2005.

Hauglustaine, D. A., Brasseur, G. P., Walters, S., Rasch, P. J., Müller, J.-F., Emmons, L. K., and Carroll, M. A.: MOZART, a global chemical transport model for ozone and evaluation, J. Geophys. Res., 28 291-28 335, 1998.

Höpfner, M., Oelhaf, H., Wetzel, H., Friedl-Valloon, F., Glatthor, N., Stiller, G. P., von Clarmann, T., Fischer, H., Kröger, C., and Deshler, T.: Evidence of scattering of tropospheric radiation by PSCs in mid-IR emission spectra: MIPAS-B observations and KOPRA simulations, Geophys. Res. Lett., 29, 1278, doi:10.1029/2001GL014443, 2002.

Höpfner, M., Luo, B. P., Massoli, P., Cairo, F., Spang, R., Snels, M., Di Donfrancesco, G., Stiller, G., v. Clarmann, T., Fischer, H., and Biermann, U.: Spectroscopic evidence for $\beta$-NAT, STS and ice in MIPAS infrared limb emission measurements of polar stratospheric clouds, Atmos. Chem. Phys., 6, 1201-1219, 2005, 
http://www.atmos-chem-phys.net/6/1201/2005/.

Houghton, J. T.: The Physics of Atmosphere, 2nd ed., Cambridge University, Cambridge, UK, 1986.

Irion, F. W., Gunson, M. R., Toon, G. C., Chang, A. Y., Eldering, A., Mahien, E., Manney, G. L., Michelsen, H. A., Moyer, E. J., Newchurch, M. J., Osterman, G. B., Rinsland, C. P., Salawitch, R. J., Sen. B., Yung, Y. L., and Zander, R.: atmospheric Trace Molecule Spectroscopy (ATMOS) experiment version 3 data retrievals, Appl. Opt., 41, 6968-6979, 2002.

Kalman, R. E.: Algebraic aspects of the generalized inverse of a rectangular matrix, Proc. of Advanced Seminar on Generalized Inverse and Applications, edited by: Nashed, M. Z., Academic, San Diego, Calif., 111-124, 1976.

Kleinert, A., Aubertin, G., Perron, G., Birk, M., Wagner, G., Hase, F., Nett, H., and Poulin, R.: MIPAS Level1B algorithms overview: operational processing and characterization, Atmos. Chem. Phys. Discuss., 6, 10 673-10 711, 2006.

Kutepov, A., Feofilov, A., Marshall, B. T., Gordley, L. L., Pesnell, W. D., Goldberg, R. A., Russell, J. M., et al.: Sensitivity of IR Temperature Retrievals in the Polar Summer MLT to NLTE considerations, Eos Trans. AGU, 87(36), Jt. Assem. Suppl., Abstract SA53A-07, 2006.

Levenberg, K.: A method for the solution of certain problems in least squares, Quart. Appl. Math., 2, 164-168, 1944.

López-Puertas, M., Funke, B., García-Comas, M., López-Valverde, M. A., and Martín-Torres, J.: Advanced MIPAS Level 2 Data Analysis (AMIL2DA), Project EVG1-CT-1999-00015, Report on the Climatology of Vibrational Temperatures, March 2002.

López-Puertas, M., Clarmann, T., Dudhia, A., Flaud, J.-M., Funke, B., García-Comas, M., Gil-López, S., Glatthor, N., Grabowski, U., Jay, V., Kiefer, M., López-Valverde, M. A., Perron, G., and Stiller, G.: Non-LTE studies for the validation of MIPAS/Envisat L2 products, Proc. Envisat Validation Workshop, ESA SP-531, Noordwijk, 2003.

López-Puertas, M., Koukouli, M. E., Funke, B., Gil-López, S., Glatthor, N., Grabowski, U., Stiller, G. P., and Clarmann, v. T.: Evidence for $\mathrm{CH} 47.6 \mu \mathrm{m}$ non-local thermodynamic equilibrium emission in the mesosphere, Geophys. Res. Lett., 32, L04805, doi:10.1029/2004GL021641, 2005.

Marquardt, D. W.: An algorithm for the least-squares estimation of non-linear parameters, SIAM J. Soc. Appl. Math., 11, 431, 1963.

Mencaraglia, F., Bianchini, G., Boscaleri, A., Carli, B., Ceccherini, S., Raspollini, P., Perrin, A., and Flaud, J.-M.: Validation of MIPAS satellite measurements of HNO3 with comparison of rotational and vibrational spectroscopy, J. Geophys. Res., 111, D19305, doi:10.1029/2005JD006099, 2006.

Menke, W.: Geophysical Data Analysis: Discrete Inverse Theory, Academic, San Diego, California, 1984.

Nash, E. R., Newmann, P. A., Rosenfield, J. E., and Schoeberl, M. R.: An objective determination of the polar vortex using Ertel's potential vorticity', J. Geophys. Res., 1001, 9471-9478, 1996.

Nett, H. and Perron, G.: ENVISAT-MIPAS: instrument commissioning \& early results, Geoscience and Remote Sensing Symposium, 2002, IGARSS '02, 2002 IEEE International, 1, 602-604, 2002a.

Nett, H., Perron, G., Sanchez, M., Burgess, A., and Mosner, P.: MIPAS In-Flight Calibration and Processor Verification, Proceedings of ENVISAT Calibration Review, Nordwick, ESA-ESTEC, ESA Publication SP-520, 2002b.
Norton, R. H. and Beer, R.: New apodizing functions for Fourier spectroscopy, J. Opt. Soc. Am., 66, 259-264, 1976; errata corrige J. Opt. Soc. Am., 67, 419, 1977.

Piccolo, C., Carli, B., and Ceccherini, S.: Test of the MIPAS retrieval code with ATMOS measurements, J. Atmos. Oceanic Technol., 21(10), 1557-1565, 2004.

Raspollini, P. and Ridolfi, M.: Mapping of Temperature and Lineof-Sight Errors in Constituent Retrievals for MIPAS/ENVISAT Measurements, Atmos. Environ., 34, 5329-5336, 2000.

Remedios, J. J.: Extreme Atmospheric Constituent Profiles for MIPAS, Proceedings of the European symposium on atmospheric measurements from space, ESTEC, Netherlands, 20-22 January, 2, 779-783, 1999.

Ridolfi, M.: Characterisation of MIPAS Line of Sight (LOS) pointing error, TN contract ESA ESRIN no. 17580, 2005.

Ridolfi, M., Carli, B., Carlotti, M., von Clarmann, T., Dinelli, B. M., Dudhia, A., Flaud, J.-M., Höpfner, M., Morris, P. E., Raspollini, P., Stiller, G., and Wells, R. J.: Optimized forward model and retrieval scheme for MIPAS near-real-time data processing, Appl. Opt., 39, 1323-1340, 2000.

Ridolfi, M., Ceccherini, S., and Carli, B.: Optimal interpolation method for intercomparison of atmospheric measurements, Opt Lett., 31, 855-857, 2006.

Rodgers, C. D.: Inverse methods for atmospheric sounding - Theory and practise, edited by: Taylor, F. W., World Scientific, 2000.

Rodgers, C. D. and Connor, B. J.: Intercomparison of remote sounding instruments, J. Geophys. Res., 108, 4116-4130, 2003.

Rosenkranz, P. W.: Shape of the 5 mmoxygen band in the atmosphere, IEEE Trans. Antennas Prop. AP-23, 498-506, 1975.

Rothman, L. S., Rinsland, C. P., Goldman, A., Massie, S. T., Edwards, D. P., Flaud, J.-M., Perrin, A., Camy-Peyret, C., Dana, V., Mandin, J.-Y., Schroeder, J., McCann, A., Gamache, R. R., Wattson, R. B., Yoshino, K., Chance, K. V., Jucks, K. W., Brown, L. R., Nemtchinov, V., and Varanasi, P.: The HITRAN Molecular Spectroscopic Database and HAWKS (HITRAN Atmospheric WorKStation): 1996 Edition, J. Quant. Spectr. Radiat. Trans., 60, 665, 1998.

Sivia, D. S.: Data analysis. A Bayesian tutorial, Clarendon Press Oxford, 1998.

Spang, R., Reise, M., and Offermann, D.: CRISTA-2 observations of the south polar vortex in winter 1997: a new dataset for polar process studies, Geophys. Res. Lett., 28, 3159-3162, 2001.

Spang, R., Eidmann, G., Riese, M., Preusse, P., Offermann, D., Pfister, L., and Wang, P. H.: CRISTA observations of cirrus clouds around the tropopause, J. Geophys. Res., 107(D23), 8174, doi:10/1029/2002JD000698, 2002.

Spang, R. and Remedios, J. J.: Observations of a distinctive infrared spectral feature in the atmospheric spectra of polar stratospheric clouds measured by the CRISTA instrument, Geophys. Res. Lett., 30(16), 1875, doi:10.1029/2003GL017231, 2003.

Spang R., Remedios, J. J., and Barkley, M. P.: Colour indices for the detection and differentiation of cloud types in infra-red limb emission spectra, Adv. Space Res., 33, 7, 1041-1047, 2004.

Verronen, P. T., Kyrölä, E., Tamminen, J., Funke, B., Gil-López, S., Kaufmann, M., López-Puertas, M., Clarmann, T. v., Stiller, G., Grabowski, U., and Höpfner, M.: A comparison of nighttime GOMOS and MIPAS ozone profiles in the stratosphere and mesosphere, Adv. Space Res., 36, 958-966, 2005.

von Clarmann, T., Dudhia, A., Echle, G., Flaud, J.-M., Harrold, 
C., Kerridge, B., Koutoulaki, K., Linden, A., Lopez-Puertas, M., Lopez-Valverde, M. A., Martin-Torres, F. J., Reburn, J., Remedios, J., Rodgers, C. D., Siddans, R., Wells, R. J., and Zaragoza, G.: Study on the simulation of atmospheric infrared spectra, Tech. Rep., Final Report of ESA Contract 12054/96/NL/CN, European Space Agency, ESTEC, Noorwijk, The Netherlands, 1998.

von Clarmann., T., Ceccherini, S., Doicu, A., Dudhia., A., Funke, B., Grabowski, U., Hilgers, S., Jay, V., Linden, A., LopezPuertas, M., Martin-Torres, F. J., Payne, V., Reburn, J., Ridolfi, M., Schreier, F., Schwarz, G., Siddans, R., and Steck, T.: A blind test retrieval experiment for infrared limb emission spectroscopy, J. Geophys. Res., 108(D23), 4746, doi:10.1029/2003JD003835, 2003a. von Clarmann, T., Glatthor, N., Grabowski, U., Höpfner, M., Kellmann, S., Kiefer, M., Linden, A., Mengistu Tsidu, G., Milz, M., Steck, T., Stiller, G. P., Wang, D. Y., Fischer, H., Funke, B., GilLópez, S., and López-Puertas, M.: Retrieval of temperature and tangent altitude pointing from limb emission spectra recorded from space by the Michelson Interferometer for Passive Atmospheric Sounding (MIPAS), J. Geophys. Res., 108(D23), 4736, doi:10.1029/2003JD003602, 2003b.

von Clarmann, T., Höpfner, M., Funke, B., López-Puertas, M., Dudhia, A., Jay, V., Schreier, F., Ridolfi, M., Ceccherini, S., Kerridge, B. J., Reburn, J., Siddans, R., and Flaud, J.-M.: Modelling of atmospheric mid-infrared radiative transfer: the AMIL2DA algorithm intercomparison experiment, J. Quant. Spectr. Radiat. Trans., 78(3-4), 381-407, 2003c. 